\section{Measurements and simulations of microtubule growth imply strong longitudinal interactions and reveal a role for GDP on the elongating end}

\author{
Joseph M. Cleary ${ }^{1}$, Tae Kim², Annan S. I. Cook ${ }^{1}$, William O. Hancock ${ }^{1, *}$, Luke M. Rice, ${ }^{2 *}$ \\ ${ }^{1}$ Department of Biomedical Engineering, Pennsylvania State University, University Park, PA \\ 16802, USA \\ ${ }^{2}$ Departments of Biophysics and Biochemistry, UT Southwestern Medical Center, 5323 Harry \\ Hines Blvd., Dallas TX 75390 \\ * Corresponding author
}


Introduction

The microtubule cytoskeleton is essential for the proper organization of the interior of eukaryotic cells: microtubules build the mitotic spindle that mediates faithful chromosome segregation during cell division (Prosser and Pelletier, 2017), they mediate organelle positioning and help establish cellular polarity, and they form tracks for motor-based transport (Desai and Mitchison, 1997). Microtubules are dynamic, multi-stranded polymers that grow and shrink by addition and loss of $\alpha \beta$-tubulin subunits from the ends of the hollow, cylindrical polymer (Cleary and Hancock, 2021; Desai and Mitchison, 1997; Gardner et al., 2013; Howard and Hyman, 2003). The dynamic properties of microtubules are central to their function, and result from the structural and biochemical properties of individual $\alpha \beta$-tubulin subunits and how they interact with the microtubule lattice. It has been challenging to define the quantitative mechanisms of microtubule dynamics, because the microtubule end is a complex biochemical environment where individual tubulins adopt different conformations and can contact variable numbers of neighboring subunits (Brouhard and Rice, 2018; Gudimchuk and McIntosh, 2021). Consequently, there is yet to be a consensus about the fundamental mechanisms governing microtubule growing, shrinking, and switching. This lack of understanding in turn limits our ability to understand how microtubule regulatory factors and/or microtubule-targeting drugs modulate polymerization dynamics.

In prior work, using a combination of nanogold-labeled yeast $\alpha \beta$-tubulin and interferometric scattering (iSCAT) microscopy (Ortega-Arroyo and Kukura, 2012; Young and Kukura, 2019), we showed that interactions between $\alpha \beta$-tubulin and the MT end could be observed at the singlemolecule level and quantified with high temporal resolution (Mickolajczyk et al., 2019b). Those measurements revealed that the association and dissociation of individual $\alpha \beta$-tubulins occurred more slowly than had been expected based on an earlier analysis of microtubule growth rates and

63 fluctuations (Gardner et al., 2011). However, the extent to which these findings were in conflict

64 was difficult to assess: in addition to using different methods to determine growth fluctuations,

65 the two studies used different sources of $\alpha \beta$-tubulin (fungal for the single molecule

66 measurements, mammalian brain for the study of fluctuations). 
68 In the present study, we used interference reflection microscopy (IRM) (Mahamdeh and Howard,

69 2019; Mahamdeh et al., 2018) to observe the in vitro growth rates and fluctuations of bovine

70 brain microtubules at high temporal resolution. We performed measurements in the presence of

71 GTP (where the microtubules can undergo catastrophe as a consequence of GTPase activity) and

72 in the presence of the hydrolysis-resistant GTP analog GMPCPP (to provide biochemically

73 simpler measurements for analysis). We fit a computational model (Ayaz et al., 2014; Kim and

74 Rice, 2019; Mickolajczyk et al., 2019b; Piedra et al., 2016) to the resulting measurements to

75 provide insight into the molecular interactions governing microtubule elongation. Microtubules

76 growing with GMPCPP showed a very low apparent critical concentration, reflecting high

77 affinity interactions between $\alpha \beta$-tubulin subunits and the microtubule end. Fluctuations in the

78 growth rates of GMPCPP microtubules were extremely low at all concentrations tested. Fitting a

79 computational kinetic model to the data revealed that the GMPCPP measurements were best

80 described by slow assembly kinetics, such that the rate of microtubule elongation is primarily

81 limited by the rate of $\alpha \beta$-tubulin associations.

83 We found that microtubules growing with GTP displayed substantially larger fluctuations in

84 growth rate than would be expected from extrapolation of the GMPCPP measurements. The

85 "GTP only" model of microtubule elongation that was able to recapitulate the GMPCPP

86 measurements could not account for the larger growth fluctuations observed with GTP.

87 However, when it allowed for GTP hydrolysis, the model produced fluctuations in growth rates

88 comparable to those observed experimentally. These larger, GTPase-induced fluctuations in

89 simulations resulted from transient, growth-inhibitory effects of GDP-tubulin at the end of one or

90 more protofilaments.

92 Our results provide the most comprehensive characterization of microtubule elongation in the

93 absence of GTPase activity, and provide some of the highest temporal resolution measurements

94 of microtubule elongation to date. The measurements and modeling reveal significant differences

95 in the apparent biochemistry of microtubule growth with GTP vs. GMPCPP, with stronger lattice

96 contacts ( $\sim 10$-fold higher affinity) in the presence of GMPCPP. Both sets of measurements can

97 be explained by biochemical models featuring relatively slow association and dissociation

98 kinetics, in which strong head-to-tail interactions between $\alpha \beta$-tubulins dictate more ragged and 
99 tapered microtubule end configurations. The modeling demonstrates that the much larger growth

100 fluctuations of microtubules with GTP are caused by intermittent 'protofilament poisoning' that

101 results from transient exposure of GDP-bound $\alpha \beta$-tubulin subunits on the microtubule end.

102 Collectively, these results provide new ideas about the fundamental mechanisms that dictate

103 microtubule growth rates and fluctuations, with implications for how regulatory factors could

104 control these processes.

105

\section{Results}

When GTPase activity is blocked, microtubules show very low fluctuations in growth rate

We used interference reflection microscopy (IRM) (Mahamdeh and Howard, 2019; Mahamdeh

111 et al., 2018) to measure the dynamics of bovine brain microtubules with high temporal resolution

112 (10 frames per second) and good signal-to-noise ratio (exceeding 4). To provide templates for

113 microtubule elongation, our assay used biotinylated, double-cycled GMPCPP 'seeds' attached to

114 a cover slip using neutravidin (Chen et al., 2019)(Fig1A; inset shows a representative image of a

115 microtubule). To measure the position of microtubule ends over time with sub-pixel resolution,

116 we adapted a previously described algorithm that fits the decay in image intensity at the

117 microtubule tip (Demchouk et al., 2011; Gardner et al., 2011; Prahl et al., 2014)(Figure 1- figure 118 supplement 1).

120 Our first series of measurements used the hydrolysis-resistant GTP analog GMPCPP to measure

121 the concentration-dependence of microtubule growth rates and fluctuations in a biochemically

122 simple setting, without complications associated with GTPase activity. Representative

123 kymographs and 'tracks' of microtubule length as a function of time at the low $(0.5 \mu \mathrm{M})$ and

124 high $(1.5 \mu \mathrm{M})$ ends of the tubulin concentration range are shown in Figure 1B; the high limit was

125 chosen to avoid conditions where microtubules formed spontaneously. We measured growth

126 rates from a large number of tracks at each tubulin concentration (Figure 1C shows the resulting

127 average growth rates), obtaining a range comparable to that observed in a prior study (Gardner

128 et al., 2011). The measured growth rates followed the expected linear relationship between mean

129 growth rate and tubulin concentration, yielding an apparent on-rate constant for microtubule 
130 binding $\left(\mathrm{k}_{\mathrm{on}}{ }^{\mathrm{app}}\right.$, slope) of $3.1 \mu \mathrm{M}^{-1} \mathrm{~S}^{-1}$ and apparent critical concentration (x-intercept) of $\sim 100$

131 nM.

133 The label-free IRM imaging provided long recordings and high time resolution. These rich data

134 sets allowed us to analyze fluctuations in microtubule growth rates at the level of individual

135 microtubules, rather than having to concatenate shorter recordings from multiple microtubules,

136 as was done in previous work (Gardner et al., 2011). To ensure that the fluctuations reported

137 were solely from microtubule growth, we used fiduciary marks on the slide to remove

138 contributions from sub-pixel stage drift (Figure 1 - figure supplement 1)(Leduc et al., 2007).

139 Analyzing microtubule growth fluctuations of individual growth episodes provided better insight

140 into the range of growth properties compared to the analysis of concatenated growth episodes

141 (Figure 1 - figure supplement 2). Because separating the growth rate from growth fluctuations

142 can be difficult when growth lengths are relatively short, as for our GMPCPP data, we evaluated

143 two methods for quantifying fluctuations around the mean growth rate (Figure 1 - figure

144 supplement 3). One method obtains growth rates and fluctuations concurrently by fitting mean-

145 squared displacement (MSD) vs time curves (Gardner et al., 2011), while the other determines

146 growth rates and fluctuations independently by analyzing the mean and variance of incremental

147 length change distributions, sampled at different time intervals (Castle et al., 2019). Separate

148 determination of growth rate and variance provided the most robust results (Figure 1 - figure

149 supplement 3).

151 Our analysis reveals that microtubule growth fluctuations in GMPCPP were very small, ranging

152 from $2-4 \mathrm{~nm}^{2} / \mathrm{s}$. These values are $\sim 5$-fold smaller than those measured in a prior study that used

153 MSD fitting to analyze fluorescence images (Gardner et al., 2011); likely causes of the

154 discrepancy and implications of the smaller fluctuations for mechanisms of microtubule

155 dynamics are addressed in the Discussion and Figure 1 - figure supplements 1-3. The

156 measurement noise in our assay was estimated to be $1.2 \mathrm{~nm}^{2} / \mathrm{s}$ by extrapolating the growth

157 variance to zero tubulin concentration. Similarly, measuring the end position of a static

158 GMPCPP and taxol-stabilized microtubule seed yielded a growth variance of $0.97 \mathrm{~nm}^{2} / \mathrm{s}$. This

159 contribution from noise means that our measurements provide an upper limit on the fluctuations 
160 around the average microtubule growth rate - the actual fluctuations are likely smaller than the

161 values we report.

162

163 We estimated the length of the microtubule end taper by quantifying the fall off in image

164 intensity at the end of a growing microtubule (Gardner et al., 2011; Maurer et al., 2014). To

165 calibrate our taper measurements, we used the known characteristics of our microscope to

166 simulate images of synthetic microtubules with defined taper lengths and then fit those images to

167 quantify the fall off in intensity at the end (Figure 1 - figure supplement 4 ). The minimal taper

168 length that was distinguishable above the point spread function of the microscope was $265 \mathrm{~nm}$

169 ( 33 tubulin), similar to (Maurer et al., 2014). We found that with increasing tubulin

170 concentrations, the fraction of time that end tapers were detectable above the measurement

171 threshold increased (Figure 1 - figure supplement 4), and that tapers alternately grew and shrank,

172 switching on timescales of 10 s of seconds (Figure 1 - figure supplement 4 ).

A minimal computational model can largely recapitulate measured growth rates and fluctuations

176 To obtain insight into the biochemistry underlying microtubule growth rates and fluctuations in

177 the presence of GMPCPP, we applied a minimal kinetic model that simulates microtubule

178 elongation at the level of individual association and dissociation events. This model, which has

179 been described previously (Ayaz et al., 2014; Kim and Rice, 2019; Mickolajczyk et al., 2019b;

180 Piedra et al., 2016) and which is similar to other biochemical models (Gardner et al., 2011;

181 Margolin et al., 2012; VanBuren et al., 2002), is summarized in Figure 2 - figure supplement 1.

182 The two parameters that determine growth rate in the model (Figure 2A; Figure 2 - figure

183 supplement 1) are the strengths of longitudinal and 'corner' (one longitudinal + one lateral)

184 interactions between $\alpha \beta$-tubulin and the lattice. The rate of $\alpha \beta$-tubulin:microtubule associations

185 in the model is determined by the bimolecular association rate constant $\mathrm{k}_{\text {on }}$ and the concentration

186 of $\alpha \beta$-tubulin (via $\mathrm{k}_{\mathrm{on}} *\left[\alpha \beta\right.$-tubulin]). Dissociation rates for different sites are calculated from $\mathrm{K}_{\mathrm{D}}$,

187 the equilibrium dissociation constant for that site (via $\left.\mathrm{k}_{\mathrm{on}} * \mathrm{~K}_{\mathrm{D}}\right)$. The experiments do not directly

188 determine the association rate constant, so we tested a range of values when trying to fit the

189 model to data. We also tested a number of different corner affinities $\left(\mathrm{K}_{\mathrm{D}}{ }^{\text {corner }}\right)$ for each $\mathrm{k}_{\text {on. }}$. For 
190 each combination of $\mathrm{k}_{\text {on }}$ and $\mathrm{K}_{\mathrm{D}}{ }^{\text {corner }}$ we optimized the longitudinal affinity $\left(\mathrm{K}_{\mathrm{D}}{ }^{\text {long }}\right)$ to obtain the

191 best fit to experimental data.

193 We found that the $\alpha \beta$-tubulin on- and off-rate constants in GMPCPP cannot be uniquely

194 constrained by fitting the microtubule growth rates alone. As long as the corner affinity is

195 sufficiently high $\left(\mathrm{K}_{\mathrm{D}}{ }^{\text {corner }}\right.$ less than $\left.\sim 100 \mathrm{nM}\right)$, the model can recapitulate the concentration-

196 dependent growth rates in GMPCPP for assumed $\mathrm{k}_{\text {on }}$ values spanning $\mathrm{a} \sim 10$-fold range (from

1970.27 to $2.2 \mu \mathrm{M}^{-1} \mathrm{~s}^{-1}$ ) (Figure 2 - figure supplement 2; selected conditions are shown in Figure

198 2B). Longitudinal interactions are predicted to be strongest ( $\mathrm{K}_{\mathrm{D}}^{\text {long }} \sim 1 \mu \mathrm{M}$ affinity or stronger)

199 for the smallest $\mathrm{k}_{\text {on }}$ value tested. Under conditions of high longitudinal affinity, pure longitudinal

200 associations onto a protofilament are relatively long-lived and consequently most of them

201 become incorporated into the growing microtubule. Conversely, longitudinal interactions are

202 predicted to be weakest ( $\mathrm{K}_{\mathrm{D}}$ long $\sim 10 \mathrm{mM}$ affinity or weaker) for the highest $\mathrm{k}_{\text {on }}$ value tested.

203 Under conditions of low longitudinal affinity, most pure longitudinal associations onto a

204 protofilament are relatively short-lived and do not contribute meaningfully to elongation. This

205 inverse relationship between assumed $\mathrm{k}_{\text {on }}$ and $\mathrm{K}_{\mathrm{D}}{ }^{\text {long }}$ holds for intermediate choices of $\mathrm{k}_{\mathrm{on}}$ and

206 yields different degrees of end tapering (Figure 2 - figure supplement 4) (Ayaz et al., 2014;

207 Cleary and Hancock, 2021; VanBuren et al., 2002).

Different choices of $\mathrm{k}_{\mathrm{on}}$ (with their correspondingly different longitudinal affinities) yielded

210 different magnitude fluctuations in growth rate that were evident in the relative divergence of

211 length vs time traces (Figure 2 - figure supplement 3). Quantifying the fluctuations from 50

212 simulated growth episodes revealed a bell-shaped response in which intermediate values of $\mathrm{k}_{\mathrm{on}}$

213 produced the largest fluctuations in growth rate (Figure 2D). To compare the (noise-free)

214 fluctuations obtained from the simulations to those measured in experiments, we added to the

215 simulated values the 'noise offset' of $0.97 \mathrm{~nm}^{2} / \mathrm{s}$ obtained from measuring the fluctuations of

216 GMPCPP seeds that were not detectably elongating. With this correction, the simulations using

217 different combinations of $\mathrm{k}_{\text {on }}, \mathrm{K}_{\mathrm{D}}{ }^{\text {corner }}$, and $\mathrm{K}_{\mathrm{D}}{ }^{\text {long }}$ produce fluctuations in growth rate that can be

218 close to those measured experimentally (Figure 2E). 
220 The model was able to recapitulate measured growth rates using a variety of assumed $\mathrm{k}_{\mathrm{on}}$ values.

221 To better define the parameters required to reproduce the measurements, we used the observed

222 growth rate fluctuations as an additional fitting constraint. The optimal parameters that emerged

223 from this more constrained fitting are: $\mathrm{k}_{\mathrm{on}}=0.74 \mu \mathrm{M}^{-1} \mathrm{~s}^{-1}, \mathrm{~K}_{\mathrm{D}}{ }^{\text {corner }}=25 \mathrm{nM}$, and $\mathrm{K}_{\mathrm{D}}{ }^{\text {long }}=87 \mu \mathrm{M}$

224 (Figure 2F). These parameters give rise to microtubule end structures that are somewhat tapered

225 (Figure 2 - figure supplement 5), but less tapered than observed experimentally (Figure 1 -

226 figure supplement 5).

Fluctuations in microtubule growth rate are substantially increased in the presence of GTP

230 To determine whether and how GTP hydrolysis affected growth rates and fluctuations, we

231 analyzed 'dynamic' microtubules growing with GTP. We measured growth rates and fluctuations

232 as described above, but using higher concentrations of tubulin because sustained microtubule

233 elongation with GTP did not occur below a tubulin concentration of $\sim 6 \mu \mathrm{M}$. Representative

234 kymographs and tracks of microtubule length as a function of time are shown in Figure $3 \mathrm{~A}$ for

235 the low $(7.5 \mu \mathrm{M})$ and high $(17.5 \mu \mathrm{M})$ concentrations used.

Average growth rates for the dynamic microtubules (Figure 3B) are roughly 10-fold greater than

238 for the GMPCPP measurements (Figure 1), mirroring the higher tubulin concentration. A linear

239 fit to the growth rates yields a $\mathrm{k}_{\text {on }}{ }^{\text {app }}$ of $2.3 \mu \mathrm{M}^{-1} \mathrm{~s}^{-1}$, close to what we obtained for GMPCPP

$240 \quad\left(\mathrm{k}_{\mathrm{on}}{ }^{\text {app }}\right.$ of $3.1 \mu \mathrm{M}^{-1} \mathrm{~s}^{-1}$, see Figure 1C). The apparent critical concentration (x-intercept) for

241 microtubule growth of $3.6 \mu \mathrm{M}$ with GTP is about 35-fold greater than for growth with GMPCPP

242 ( $\sim 100 \mathrm{nM}$, Figure 1C), reflecting weaker interactions of tubulin with the microtubule end in the

243 presence of GTP. The measured growth rates and the differences between growth rates with GTP

244 or GMPCPP are consistent with prior work (Gardner et al., 2011).

246 The fluctuations in growth rate for microtubules growing with GTP are several-fold larger than

247 those obtained with GMPCPP, and depend 3-fold more steeply on concentration (slope of 5.6

$248 \mathrm{~nm}^{2} \mathrm{~s}^{-1} / \mu \mathrm{M}$ for GTP, Figure 3B; slope of $1.9 \mathrm{~nm}^{2} \mathrm{~s}^{-1} / \mu \mathrm{M}$ for GMPCPP, Figure 1C). Notably, the

249 magnitude of fluctuations in GTP is $\sim 7$-fold smaller than reported in a prior study that used a

250 different method to analyze fluctuations (Gardner et al., 2011), even though measured growth 
251 rates were comparable between the two studies. Because the growth rates in GTP are

252 considerably faster than in GMPCPP, it is not clear how much the increased fluctuations reflect

253 real differences in growth properties or simply the faster overall growth rates. To provide a better

254 way to compare the magnitude of fluctuations between the two conditions, we plotted the

255 measured fluctuations as a function of the corresponding microtubule growth rates (Figure 3C).

256 Extrapolating the GMPCPP fluctuations out to faster growth rates reveals that microtubules

257 growing with GTP fluctuate substantially more around their average growth rate than

258 microtubules growing with GMPCPP, even accounting for different growth rates.

In the computational model, transient protofilament poisoning by GDP can explain the increased growth fluctuations of dynamic microtubules

What is the biochemical origin of increased microtubule growth fluctuations in the presence of GTP? We used the computational model to begin addressing this question. We fit the model to 265 the concentration dependent GTP growth rates, initially ignoring GTP hydrolysis to mirror how 266 we analyzed the GMPCPP data. We used the optimal $\mathrm{k}_{\text {on }}$ determined from the GMPCPP analysis $267\left(0.74 \mu \mathrm{M}^{-1} \mathrm{~s}^{-1}\right)$ and tested a range of corner affinities. The corner affinity that best fit the GTP growth rates was $\mathrm{K}_{\mathrm{D}}{ }^{\text {corner }}=2.9 \mu \mathrm{M}$ (Figure $4 \mathrm{~A}$ ), roughly 100-fold weaker than for the GMPCPP data and consistent with the large change in apparent critical concentration. The longitudinal

\section{GMPCPP.}

While the "GTPase-free" model was able to fit the observed growth rates, it dramatically

274 underestimated both the magnitude of measured fluctuations and their concentration-dependence

275 (Figure 4B). Altering the model (see Methods) to use different on-rate constants for longitudinal, 276 corner, and 'bucket' binding sites (Castle and Odde, 2013; Gardner et al., 2011) did not improve 277 predictions of fluctuations (Figure 4 - figure supplement 1). Thus, in contrast to what we

278 observed for elongation in GMPCPP, the GTPase-free model cannot recapitulate the growth

279 fluctuations measured in GTP. Instead, some other mechanism or state that is missing from the 280 minimal model must be needed to generate larger fluctuations. 
282 We simulated microtubule dynamics in the presence of GTPase activity to test whether GTPase

283 could represent this missing, fluctuation-increasing mechanism. Incorporating GTPase activity

284 required two additional parameters ((Kim and Rice, 2019; Piedra et al., 2016; VanBuren et al.,

285 2002); see Methods): the rate constant for GTP hydrolysis, and the weakening effect of GDP on

286 the strength of tubulin:tubulin interactions (Figure 4C). As the rate of GTPase activity was

287 increased in successive simulations, microtubule growth rates decreased and their fluctuations

288 increased (Figure 4D). It is notable and somewhat counterintuitive that the fluctuations

289 increased as growth rates decreased, because slower growth would normally be expected to

290 produce smaller fluctuations (as seen in Figure 3C). The magnitude of the fluctuations increased

291 sufficiently to match or even exceed the experimental measurements (dashed red line in Figure

292 4D). Thus, GTPase activity and its introduction of a second biochemical state allows the

293 simulations to recapitulate the experimentally observed magnitude of fluctuations.

295 GTPase activity cannot occur in the terminal plus-end subunits because GTPase activity requires

296 that a longitudinal interface be formed 'above' the GTP (Nogales et al., 1998). So how does

297 GTPase activity affect microtubule growth rates and fluctuations, which are dictated by the

298 binding and unbinding of subunits at the microtubule end? GDP-bound tubulins can be exposed

299 on the microtubule end as a consequence of the dissociation of more terminal subunits (closer to

300 the protofilament end). Instantaneous growth rates calculated from simulations without GTPase

301 (Figure 4E, top left) vary in a relatively narrow range, whereas those calculated from simulations

302 with GTPase (Figure 4E, top right) show much larger variations. In the simulations with

303 GTPase, the largest decreases in growth rate occur when multiple protofilaments have a terminal

304 GDP (compare Figure 4E top and bottom). Even a relatively low average frequency of GDP

305 exposure shows demonstrable effects on microtubule growth rates, and even more so on

306 fluctuations (Figure 4F).

\section{Discussion}


312 We used interference reflection microscopy (IRM) (Mahamdeh and Howard, 2019; Mahamdeh

313 et al., 2018) to measure microtubule dynamics with high time resolution and without the need for

314 fluorescent labeling, which allowed for longer recordings. The IRM imaging provided high

315 signal to noise and allowed the position of the microtubule end to be defined with sub-pixel

316 precision throughout relatively long (several minutes) time-lapse series. Whereas the growth

317 rates we measured for GMPCPP and GTP are largely consistent with prior studies (Brouhard et

318 al., 2008; Gardner et al., 2011; Vemu et al., 2017), the associated fluctuations were substantially

319 smaller than previously reported (Gardner et al., 2011). Several factors contribute to the reduced

320 magnitude fluctuations we report here: sub-pixel drift correction, a more robust method for

321 determining fluctuations, and the ability of IRM to image microtubule growth at higher frame

322 rates and long durations. The impact of these lower magnitude fluctuations on constraining

323 mechanisms of microtubule dynamics and regulation is discussed below.

Microtubule elongation in the absence of GTPase: slow associations and small fluctuations

327 Microtubules grew very steadily with only small fluctuations in growth rate when GTPase

328 activity was suppressed. Kinetic simulations implementing a minimal biochemical model for

329 microtubule elongation recapitulated the observed growth rates using a range of association rate

330 constants, with slower association rates compensated for by stronger longitudinal interactions.

331 Fluctuations around the average growth rate in the model provided stronger fitting constraints

332 because they were more sensitive to the choice of association rate constant: at comparable

333 growth rates, fluctuations were highest around the middle of the range of association rate

334 constants tested, and lower at the 'fast' and 'slow' extremes. The different characteristic

335 magnitudes of fluctuations in growth rate reflected the extent to which the microtubule end

336 configurations (number of longitudinal and corner sites) were varying in response to the different

337 strength longitudinal affinities (Figure 5).

339 In our minimal model for microtubule dynamics, the parameters that gave the best fit to the

340 GMPCPP growth rates and growth rate fluctuations corresponded to relatively slow associations

341 and relatively strong longitudinal associations. The slower, stronger longitudinal interaction

342 model that best describes our GMPCPP data is consistent with a recent single-molecule study of 
343 yeast microtubule elongation (Mickolajczyk et al., 2019b). It is also consistent with the

344 emerging view (Erickson, 2019; Gudimchuk and McIntosh, 2021; McIntosh et al., 2018) that

345 microtubule protofilaments can elongate somewhat independently of each other. This view is

346 based on cryo-ET images showing flared plus-ends (McIntosh et al., 2018) and the associated

347 mechanochemical models, and implicitly requires relatively strong longitudinal interactions.

Transient exposure of GDP-terminated protofilaments: a major contributor to fluctuations in

350 microtubule growth rate

352 Our measurements of microtubule elongation with GTP ('dynamic microtubules') showed

353 fluctuations that were larger in magnitude than expected from extrapolation of the GMPCPP

354 data. To fit the observed growth rates with a GTPase-free kinetic model required weaker

355 longitudinal and corner binding affinities compared to the GMPCPP data, reflecting the more

356 elevated critical concentration of the dynamic microtubules (growing in GTP), and revealing a

357 significant biochemical difference between GMPCPP and GTP in the interactions that drive

358 elongation. The GTPase-free model, which only considers the GTP state of tubulin, could not

359 recapitulate the growth fluctuations of dynamic microtubules. Because the GTPase-free model

360 could recapitulate both the growth rates and fluctuations of microtubules growing in GMPCPP, it

361 seemed likely that the fluctuations of dynamic microtubules reflect contributions from at least

362 one additional biochemical state. Our modeling showed that exposure of GDP-bound $\alpha \beta$-tubulin

363 on the end of protofilaments increases the variability in microtubule growth by transiently

364 'poisoning' elongation, yielding fluctuations comparable to those observed experimentally. Thus,

365 the fluctuations of growing dynamic microtubules do not solely reflect properties of GTP-

366 tubulin. The notion that growing microtubule ends expose GDP is not widely accepted, but we

367 argue that it should be considered as an important factor. Indeed, the decrease in growth rate that

368 accompanies GDP exposure(s) may represent an early event in the initiation of catastrophe

369 (Piedra et al., 2016).

\section{$371 \quad$ Limitations of the modeling}


373 We chose to use biochemically simplified models in the hopes of identifying the minimal set of 374 parameters capable of recapitulating a given set of observations. Consequently, we did not

375 attempt to explicitly account for either the assembly-dependent conformational changes that

376 accompany $\alpha \beta$-tubulin incorporation into the microtubule and that probably alter the strength of

377 lattice contacts (Brouhard and Rice, 2014; Brouhard and Rice, 2018), or for 'mechanochemical'

378 effects that consider spring-like properties of $\alpha \beta$-tubulin conformation (Brouhard and Rice, 2018;

379 Gudimchuk and McIntosh, 2021). Because we refine the longitudinal and corner affinities

380 independently (without imposing a fixed relationship between the two), some of these effects

381 might be captured implicitly. We also did not allow for the possibility of GDP to GTP exchange

382 on the microtubule end (Piedra et al., 2016). This is a lesser limitation because a finite rate of

383 exchange would only modulate the amount of GDP on the microtubule end for a given GTPase

384 rate; it would not eliminate the 'poisoning' effect of GDP exposure that increases fluctuations in

385 growth rate. Whatever the limitations of our model, our results clearly show that (i) there are

386 'excess' fluctuations for microtubules with GTP compared to GMPCPP, and (ii) a simple 'one

387 state' model can recapitulate the growth rates and fluctuations in GMPCPP, but an additional

388 state is required to generate the larger fluctuations observed with GTP.

Implications for mechanisms of dynamics and regulation

Our findings have three general implications for the mechanisms of microtubule dynamics and

393 regulation. First, they support a model in which the association kinetics are relatively slow, with

394 longitudinal associations correspondingly strong. This view is consistent with a recent single-

395 molecule study of yeast microtubule growth (Mickolajczyk et al., 2019b), and it also resonates

396 with structure-based evidence that microtubule protofilaments can elongate somewhat

397 independently from one another (so-called flared ends) (Erickson, 2019; Gudimchuk and

398 McIntosh, 2021; McIntosh et al., 2018). Second, although GDP-tubulin is not widely thought to

399 be present on growing microtubule ends, our results indicate that transient exposure of GDP-

400 tubulin on protofilament ends causes larger fluctuations in microtubule growth rate than would

401 be expected from 'all GTP' elongation. Because the size of the GTP cap decreases with slower

402 growth rates (Duellberg et al., 2016), this consequence for GDP-terminated protofilaments

403 provides a possible mechanism for the initiating events of microtubule catastrophe. Finally, our 
404 findings provide new ways of thinking about potential mechanisms that regulatory factors use to

405 control microtubule dynamics. The relatively slow association kinetics we observe imply that

406 modulating the rate of $\alpha \beta$-tubulin association or dissociation each provide viable strategies for

407 controlling microtubule elongation rates. Indeed, Stu2/XMAP215 family polymerases are

408 thought to accelerate microtubule growth by increasing the rate of $\alpha \beta$-tubulin associations (Ayaz

409 et al., 2014; Ayaz et al., 2012; Brouhard et al., 2008). More speculatively, it seems plausible that

410 a regulatory factor might be able to control microtubule elongation or catastrophe by altering the

411 lifetime of GDP-tubulin on the microtubule end, either by altering the rate of nucleotide

412 hydrolysis or exchange, or by altering the rate at which the GDP-bound subunits dissociate from

413 the growing end.

\section{Acknowledgements}

417 This study was supported by NSF MCB-1615938 and NIH R01-GM135565 to LMR, and by

418 NIH R35 GM139568 to WOH. JC received support from NIH T32 GM108563, and TK received 419 support from NIH T32 GM008297. We thank L. Ammerman for helpful critical comments on 420 the manuscript.

\section{Author contributions}

424 Conceptualization, Methodology, Software, Validation, Formal analysis, Investigation,

TK

Conceptualization, Methodology, Software, Validation, Formal analysis, Writing - review \& editing

WH

Conceptualization, Writing - review \& editing, Supervision, Project administration, Funding acquisition.

LR 


\section{Methods}

\section{Tubulin purification and labeling}

PC-grade bovine brain tubulin was purified as previously described (Uppalapati et al., 2009), cycled twice, quantified by $\mathrm{A}_{280}\left(\varepsilon=115,000 \mathrm{M}^{-1} \mathrm{~cm}^{-1}\right)$, diluted to either 80 or $15 \mu \mathrm{M}$ in BRB80 ( $80 \mathrm{mM}$ K-Pipes, $2 \mathrm{mM}$ EGTA, $2 \mathrm{mM} \mathrm{MgCl}_{2}$, $\mathrm{pH}$ 6.9), aliquoted, and flash frozen in liquid nitrogen. Aliquots were stored at $-80{ }^{\circ} \mathrm{C}$ and used within 2 months. Tubulin was biotinylated by polymerizing a solution of $40 \mu \mathrm{M}$ free tubulin, $1 \mathrm{mM} \mathrm{GTP}, 4 \mathrm{mM}$ $\mathrm{MgCl}_{2}$, and $5 \%$ DMSO for 30 minutes at $37^{\circ} \mathrm{C}$, adding an equimolar amount of EZ-Link NHSBiotin in DMSO (ThermoFisher 20217), and reacting for 30 mins at $37^{\circ} \mathrm{C}$. The microtubules were then pelleted (all pelleting was $10 \mathrm{~min}$ at 30 psi in a Beckman Airfuge), the pellet was resuspended in cold BRB80 and incubated on ice for $30 \mathrm{~min}$, spun for $10 \mathrm{~min}$ at 30 psi in a precooled rotor $\left(4^{\circ} \mathrm{C}\right)$, and the supernatant collected. The biotinylated tubulin was then subjected to another round of polymerization, pelleting, and depolymerization, and the concentration was checked using $\mathrm{A}_{280}$. The fraction of biotin-labeled tubulin was measured using a Biocytin Biotin Quantification Kit (Thermo Scientific \#44610). Final stocks were diluted using unlabeled tubulin to $40 \mu \mathrm{M}$ with a $33 \%$ biotin-labeled fraction. To make biotinylated microtubule seeds, $20 \mu \mathrm{M}$ biotinylated tubulin was mixed with $1 \mathrm{mM}$ GMPCPP and $4 \mathrm{mM} \mathrm{MgCl}_{2}$, and incubated at $37^{\circ} \mathrm{C}$ for 60 minutes to form stable microtubule seeds. The solution was then diluted to $2 \mu \mathrm{M}$ tubulin with $0.5 \mathrm{mM} \mathrm{GMPCPP}$ and $2 \mathrm{mM} \mathrm{MgCl}_{2}$ and allowed to grow at $37{ }^{\circ} \mathrm{C}$ for 5 hours to generate longer seeds. The seeds were pelleted and resuspended in BRB80 with $20 \%$ glycerol, flash frozen in liquid nitrogen, and stored for up to one month at $-80^{\circ} \mathrm{C}$. On the day of the experiments, an aliquot of seeds was thawed at $37{ }^{\circ} \mathrm{C}$ in the water bath, pelleted to remove the glycerol, resuspended in $\mathrm{BRB} 80$ with $0.5 \mathrm{mM} \mathrm{MgCl} 2$ and $0.5 \mathrm{mM}$ GMPCPP, and diluted as needed.

\section{Microtubule dynamics experiments}

Cover slips (18 x18 mm Corning) were cleaned overnight in $6 \mathrm{M} \mathrm{HCl}$, rinsed with $\mathrm{ddH}_{2} 0$, plasma cleaned (Harrick Plasma) for 12 minutes, and incubated in a vacuum-sealed desiccator with 1H,1H,2H,2H-perfluorodecyltrichlorosilane (Alfa Aesar L165804-03) overnight. Before use, the silanization was checked using a droplet test to confirm hydrophobicity.

Flow-cells were made by fixing two pieces of double-sided tape about $10 \mathrm{~mm}$ apart to an ethanol washed and $\mathrm{ddH}_{2} \mathrm{O}$ rinsed microscope slide, and covering with a silanized coverslip. $600 \mathrm{nM}$ neutravidin (ThermoFisher) was flowed into the chamber, followed by 5\% F127 (Sigma P2443$250 \mathrm{G}$ ), $2 \mathrm{mg} / \mathrm{mL}$ casein (Sigma C-7078), and biotinylated microtubule seeds at a concentration to achieve 5-10 seeds per each $60 \times 60 \mu \mathrm{m}$ field of view. Biotinylated BSA $(1 \mathrm{mg} / \mathrm{mL})$ was then added to the flow chamber to block any of the unused neutravidin on the cover slip. Finally, polymerization solution was added to the chamber, consisting of $0.05 \%$ methycellulose (Sigma M0512-100G), $1 \mathrm{mM} \mathrm{Mg-GTP} \mathrm{or} \mathrm{Mg-GMPCPP} \mathrm{(Jena} \mathrm{Biosciences),} \mathrm{and} \mathrm{an} \mathrm{oxygen} \mathrm{scavenging}$ system $(80 \mu \mathrm{g} / \mathrm{mL}$ Catalase (Sigma C1345-1G), $100 \mathrm{mM}$ DTT, $200 \mathrm{mM}$ D-Glucose (EMD Millipore Corp DX0145-1), $200 \mu \mathrm{g} / \mathrm{mL}$ Glucose Oxidase (EMD Millipore Corp 345386-10gm) in BRB80. The flow cell was then sealed using nail polish and let to sit for 5 minutes, and then placed onto the objective of the Nikon TE-2000 TIRF microscope that was pre-warmed using an objective heater to $30^{\circ} \mathrm{C}$. Once the surface was found, the chamber was allowed to equilibrate to temperature for 5 minutes. 
Microtubules were visualized using Interference Reflection Microscopy (IRM) (Mahamdeh et al., 2018) using a blue (440 nm) LED (pE-300white, CoolLED, UK) at $1.25 \%$ power. The LED was attached to the fluorescent line and the illumination NA was optimized to achieve the maximum signal to noise as shown in (Mahamdeh et al., 2018). Videos were collected at 10 frame/s for $900 \mathrm{~s}$.

\section{Video post-processing} Each video was flat-fielded to correct for uneven illumination, as follows. An out-of-focus image stack was acquired, the images averaged and converted to a 32-bit image in ImageJ (Schneider et al., 2012), and then normalized to a mean of 1 by dividing the intensity at each pixel by the average pixel intensity across the image. Flat fielding was achieved by dividing every frame of acquired videos by this background image. The flat-fielded image stacks were then inverted in ImageJ to generate light microtubules on a dark background, and converted to 8bit images for analysis. Stage drift was corrected by tracking fiduciary marks (small debris) found on the surface. Initially 10 fiduciary marks were tracked using FIESTA (Ruhnow et al., 2011), and the tracks averaged. A custom-MATLab script was built to correct for drift to the precision of one pixel. The script created a mask and centered the fiduciary marks to a given pixel on the mask throughout the movie. This pixel-corrected movie was used in subsequent analysis.

\section{Tracking microtubule growth}

Microtubule growth was measured using a previously described tracking algorithm (Demchouk et al., 2011; Prahl et al., 2014) in MATLab. In short, in the first frame of a movie, the user defines one point in the microtubule backbone and one at the microtubule tip. From these points, the software defines a region of interest around the microtubule and uses a centroid calculation to define the center point of the microtubule. Using pixel coordinates x' and y', for each pixel in x', the subpixel microtubule position in $\mathrm{y}^{\prime}$ is determined by fitting the intensity $\mathrm{I}\left(\mathrm{y}^{\prime}\right)$ to a Gaussian:

$$
I\left(y^{\prime}\right)=I_{\text {Background }}+I_{M T} e^{-\frac{\left(y^{\prime}-y_{C o M}\right)^{2}}{2 \sigma^{2}}}
$$

where $\mathrm{I}_{\text {Background }}$ is the mean background intensity, $\mathrm{I}_{\mathrm{MT}}$ is the peak intensity above background, $\mathrm{y}_{\mathrm{CoM}}$ is the center of mass of the microtubule backbone, and $\sigma$ is the Gaussian standard deviation. Once the backbone positions are defined in the $\mathrm{x}^{\prime}-\mathrm{y}^{\prime}$ coordinate system, a line is fit to the backbone and used to create a new 1D coordinate system, $\mathrm{x}^{\prime \prime}$, along the microtubule backbone. In $\mathrm{x}^{\prime \prime}$, points are defined every $58 \mathrm{~nm}$ (the pixel size in $\left.\mathrm{x}-\mathrm{y}\right)$, and an intensity value, $\mathrm{I}\left(\mathrm{x}^{\prime \prime}\right)$ is obtained by averaging the intensities of the closest five pixels in y. For each movie frame, the position of the microtubule tip, $\mu_{\mathrm{MT}}$, is calculated by fitting a Gaussian survival function:

$$
I\left(x^{\prime \prime}\right)=\frac{1}{2} I_{M T} \operatorname{erfc}\left(\frac{x^{\prime \prime}-\mu_{M T}}{\sqrt{2} \sigma_{M T}}\right)+I_{B \text { ackground }}
$$

where $\mathrm{I}_{\mathrm{Background}}$ is the mean background intensity, $\mathrm{I}_{\mathrm{MT}}$ is the peak signal above the background, 
al., 2011) through the movie, and calculating their average position, Fid( $\left.x^{\prime}, y^{\prime}\right)$. Subpixel drift caused artifacts because the microtubule length is defined as the tip position $\mu_{\mathrm{MT}}\left(\mathrm{x}^{\prime}, \mathrm{y}^{\prime}\right)$ relative a central backbone position $\mathrm{B}(\mathrm{x}, \mathrm{y})$. Because $\mathrm{B}(\mathrm{x}, \mathrm{y})$ is defined in the first frame, drift introduces an undetected error. This sub-pixel stage drift was corrected in two stages. Because the $\mathrm{x}$ position of the backbone was fixed by the pixel position, subtracting the fiduciary alone corrected for subpixel drift in $\mathrm{x}$. Thus, the corrected microtubule length relative to the central backbone position

$$
\Delta L_{x}=\mu_{M T}(x)-B(x)-F i d(x)
$$

The backbone position in $\mathrm{y}^{\prime}$ was determined by fitting $\mathrm{I}\left(\mathrm{y}^{\prime}\right)$ in every frame, such that sub-pixel movements in y were captured by the sub-pixel fit to the backbone (Eq 1). Thus, Fid(y) did not need to be considered. However, because the microtubule was at an angle $(\Theta)$ to the $x$-axis and the $\mathrm{x}$-position was set by the pixel position, subpixel drift in the $\mathrm{x}$-direction led to an error in $\mathrm{B}(\mathrm{y})$. This necessitated the following correction:

$$
\Delta L_{y}=\mu_{M T}(y)-B(y)-\sin \Theta * F i d(x)
$$

After these corrections, microtubule growth was calculated by the change of length in $\mathrm{x}$ and $\mathrm{y}$ :

$$
\Delta L=\sqrt{\Delta L_{x}^{2}+\Delta L_{y}^{2}}
$$

\section{Analysis of microtubule growth}

The tip displacement of each growing microtubule was tracked at 10 frames/s and sections of the tracks were selected that included a minimum of 90 seconds (900 points) of sustained growth with no tracking errors. Tracking errors were easily identified as large deviations from the constant growth, often for multiple frames in succession.

Tip growth fluctuations were analyzed following a method developed by Castle and Odde (Castle et al., 2019) as follows. Microtubule tip displacement traces were downsampled to different effective frame rates by taking the mean tip position of $n$ frames. This process was repeated from $n=2$ to 300 points (lag time $=0.2-30$ seconds), which created $n-1$ downsampled traces for each $\mathrm{n}$. At each effective frame rate, instantaneous length displacement distributions $(\Delta \mathrm{L})$ were collected by subtracting the position in each "frame" from the position in the previous "frame". For each lag time, the mean displacement, $\mu_{\Delta \mathrm{L}}$, and the displacement variance, $\sigma_{\Delta \mathrm{L}}{ }^{2}$, were calculated by fitting a normal to the $\Delta \mathrm{L}$ distribution.

The growth rate was calculated by fitting a line to the mean displacement at varying lag times using:

$$
\text { Growth Rate }=\frac{\mu_{\Delta L}}{\Delta t}=\frac{\mu_{\Delta L}}{n * t_{\text {exp }}}
$$

where $t_{\exp }$ is the original exposure time at 10 frames $/ \mathrm{s}(0.1 \mathrm{~s})$ and $\mathrm{n}$ is the size of the downsampling window.

The growth variance (D) was calculated by fitting the variance of the displacement at varying lag times, $\Delta t$, to a biphasic fit:

$$
\text { Growth Variance }=2 D \Delta t+\frac{\sigma_{i}^{2}}{\Delta t^{f}}
$$


At short lag times, the growth variance is dominated by experimental measurement error, $\sigma_{\mathrm{i}}$, arising from fitting the position the microtubule tip. This measurement error term, $\sigma_{\mathrm{i}}$, decays at approximately $1 / \Delta t^{1}$. The growth variance, $\mathrm{D}$, is modeled as $1 \mathrm{D}$ diffusion and is calculated by fitting a line to the Growth Variance curve using a minimum lag time of 10 seconds to allow for both phases to be measurable. The best fit was determined by the $\mathrm{R}^{2}$ value of the fit in MATLab.

\section{Model convolution of microtubule end structure}

We used model convolution (Gardner et al., 2010) to generate simulated microtubule images used for benchmarking the tip taper analysis. Briefly, microtubule backbones were generated, with an average size of $6.5 \mu \mathrm{m}$, following a 3 start helix B-lattice model. The radius of the microtubule was set to $25 \mathrm{~nm}$ and the tubulin dimer length was set to $8.2 \mathrm{~nm}$. Tapered microtubule plus-ends were designed as a uniform distribution of protofilament lengths with stepwise increases in length occurring linearly from the $1^{\text {st }}$ and $13^{\text {th }}$ protofilament, making the center protofilament the longest. The average photon emission per tubulin subunit was determined by measuring the magnitude of the microtubule signal (1160 arbitrary intensity units, corresponding to a $5.2 \%$ contrast signal) relative to the average signal from back reflected light (22300 units) on a 16-bit image (65535 units maximum) (Mickolajczyk et al., 2019a). A mask was defined and the microtubule placed randomly along a 1024 x 1024 pixel image $(59.4$ x 59.4 $\mu \mathrm{m})$. Then photons were generated and distributed to various positions along the artificial image according to the Gaussian standard deviation calculated from the full-width half maximum (250 $+/-90 \mathrm{~nm}$ ) measured using the cross-section of the microtubule backbones in the images. Photon shot noise was then added based on the standard deviation of the background (181 units out of 65535; this corresponds to a signal-to-noise of 6.2). The final image was then inverted and converted to an 8-bit image following the post-processing procedure used on the experimental videos and the microtubule tip was tracked using the same tracking algorithm (Demchouk et al., 2011; Prahl et al., 2014) used for the experimental measurements.

\section{Simulating microtubule growth}

We used the simulation code and analysis algorithms described previously (Kim and Rice, 2019; Mickolajczyk et al., 2019b; Piedra et al., 2016), with minor modifications. Briefly, (diagrammed in Figure 1 - figure supplement 1), the code performs kinetic Monte Carlo simulations of microtubule polymerization dynamics. The overall approach is similar to (VanBuren et al., 2002), with some differences described below. MT dynamics are simulated one biochemical reaction at a time ( $\alpha \beta$-tubulin subunit association or dissociation, and GTP hydrolysis), with the microtubule lattice represented by a two-dimensional array with a periodic boundary condition that mimics the cylindrical wall and 'seam' of 13 protofilament MTs. Association reactions are allowed at the end of each protofilament, and assumed to occur at a rate given by $k_{\text {on }}[\alpha \beta$-tubulin], where $k_{\text {on }}$ denotes the assumed on rate constant (but see below for a site-dependent association rate constants). Dissociation reactions occur at a rate given by $k_{\mathrm{on}} \times K_{\mathrm{D}}$, where $K_{\mathrm{D}}$ is the affinity determined by the sum of longitudinal and lateral contacts for the dissociating subunit. In practice this means that dissociations occur almost exclusively from terminal subunits. As described previously, (i) we do not allow pure lateral associations, and consequently the model parameter describing the strength of lateral contacts does not account for the entropic cost of subunit immobilization, and (ii) we do not allow completely surrounded subunits to dissociate. The GTPase reaction is assumed to occur at a rate given by $\mathrm{k}_{\text {GTPase }}$ on all non-terminal subunits. Our parameterization assumes that the nucleotide (GTP or GDP) acts in trans (Piedra et al., 
2016; Rice et al., 2008) to modulate the strength of longitudinal contacts. The modulation is represented as a multiplicative factor $X$ such that $K_{D}(G D P)=X^{*} K_{D}(G T P)$, where $X$ is a large, positive number, and $K_{D}(G D P)$ and $K_{D}(G T P)$ represent the affinity of a given interaction with GDP or GTP, respectively, at the longitudinal interface. An 'execution time' for each possible event is chosen by randomly sampling an exponential distribution $\mathrm{e}^{\text {-rate*t}}$, where rate denotes the characteristic rate for that type of event. At each step of the simulation, the fastest event (shortest execution time) is implemented, meaning that the microtubule configuration is updated, the list of possible events and rates refreshed accordingly, and the simulation time advanced by the execution time. In this way, different kinds of reactions occur with relative probability determined by their relative rates. The simulations output lists of user-specified quantities (such as the number of tubulins in the microtubule, or the lengths of each protofilament, or the number of GTP-tubulins in the microtubule, or others) as a function of time.

\section{Parameter Estimation}

We used iterative fitting in Matlab to identify model parameters that could recapitulate observed measurements of growth rates or growth rate fluctuations. Briefly, 50 independent simulations of length $600 \mathrm{~s}$ were run for a given set of parameters and using the same concentrations as for the measurements (GMPCPP: 0.5, 0.7, 1.1, 1.3, and 1.5 $\mu \mathrm{M}$; GTP: 7.5, 10.0, 12.5, 15.0, and 17.5 $\mu \mathrm{M}$ ). The discrepancy between chosen simulation outputs (normally growth rates but in some cases also fluctuations in growth rate), averaged over the independent simulations, was minimized by varying the longitudinal and corner affinities. For simulations that included GTPase activity, we first identified the 'GDP weakening factor' that gave shrinking rates for all GDP microtubules that were comparable to the measured shrinking rate.

\section{Allowing site-dependent on-rate constants} We modified the model to allow for different on-rate constants for different kinds of binding sites (longitudinal, corner, or bucket sites, which correspond to a longitudinal contact with 0,1 , or 2 lateral contacts). Site-dependent on-rate constants were previously described in (Castle and Odde, 2013), and we used similar ratios between longitudinal, corner, and bucket on-rate constants identified in that work $\left(\mathrm{k}_{\text {on }}{ }^{\text {long }} / \mathrm{k}_{\text {on }}{ }^{\text {corner }}=1.67, \mathrm{k}_{\text {on }}{ }^{\text {corner }} / \mathrm{k}_{\text {on }}{ }^{\text {bucket }}=4.62\right)$. We set the association rate constant into corner sites to be identical to that used in the simpler, single association rate constant simulations, and then scaled the longitudinal and bucket associations to be faster and slower, respectively. This way of doing things minimized the differences in the strength of longitudinal and corner interactions between the single and multiple association rate constant simulations. Fitting the simulations to the measurements was performed as described above. 


\section{References}

Ayaz, P., S. Munyoki, E.A. Geyer, F.A. Piedra, E.S. Vu, R. Bromberg, Z. Otwinowski, N.V. Grishin, C.A. Brautigam, and L.M. Rice. 2014. A tethered delivery mechanism explains the catalytic action of a microtubule polymerase. elife. 3:e03069.

Ayaz, P., X. Ye, P. Huddleston, C.A. Brautigam, and L.M. Rice. 2012. A TOG:alphabeta-tubulin complex structure reveals conformation-based mechanisms for a microtubule polymerase. Science. 337:857-860.

Bohner, G., N. Gustafsson, N.I. Cade, S.P. Maurer, L.D. Griffin, and T. Surrey. 2016. Important factors determining the nanoscale tracking precision of dynamic microtubule ends. $J$ Microsc. 261:67-78.

Brouhard, G.J., and L.M. Rice. 2014. The contribution of alphabeta-tubulin curvature to microtubule dynamics. J Cell Biol. 207:323-334.

Brouhard, G.J., and L.M. Rice. 2018. Microtubule dynamics: an interplay of biochemistry and mechanics. Nat Rev Mol Cell Biol. 19:451-463.

Brouhard, G.J., J.H. Stear, T.L. Noetzel, J. Al-Bassam, K. Kinoshita, S.C. Harrison, J. Howard, and A.A. Hyman. 2008. XMAP215 is a processive microtubule polymerase. Cell. 132:79-88.

Castle, B.T., and D.J. Odde. 2013. Brownian dynamics of subunit addition-loss kinetics and thermodynamics in linear polymer self-assembly. Biophys J. 105:2528-2540.

Castle, B.T., D.J. Odde, and D.K. Wood. 2019. Rapid and inefficient kinetics of sickle hemoglobin fiber growth. Sci Adv. 5:eaau1086.

Chen, G.Y., J.M. Cleary, A.B. Asenjo, Y. Chen, J.A. Mascaro, D.F.J. Arginteanu, H. Sosa, and W.O. Hancock. 2019. Kinesin-5 Promotes Microtubule Nucleation and Assembly by Stabilizing a Lattice-Competent Conformation of Tubulin. Curr Biol. 29:2259-2269 e2254.

Cleary, J.M., and W.O. Hancock. 2021. Molecular mechanisms underlying microtubule growth dynamics. Curr Biol. 31:R560-R573.

Demchouk, A.O., M.K. Gardner, and D.J. Odde. 2011. Microtubule Tip Tracking and Tip Structures at the Nanometer Scale Using Digital Fluorescence Microscopy. Cell Mol Bioeng. 4:192-204.

Desai, A., and T.J. Mitchison. 1997. Microtubule polymerization dynamics. Annu Rev Cell Dev Biol. 13:83-117.

Duellberg, C., N.I. Cade, D. Holmes, and T. Surrey. 2016. The size of the EB cap determines instantaneous microtubule stability. eLife. 5.

Erickson, H.P. 2019. Microtubule Assembly from Single Flared Protofilaments-Forget the Cozy Corner? Biophys J. 116:2240-2245.

Gardner, M.K., B.D. Charlebois, I.M. Janosi, J. Howard, A.J. Hunt, and D.J. Odde. 2011. Rapid microtubule self-assembly kinetics. Cell. 146:582-592.

Gardner, M.K., B.L. Sprague, C.G. Pearson, B.D. Cosgrove, A.D. Bicek, K. Bloom, E.D. Salmon, and D.J. Odde. 2010. Model Convolution: A Computational Approach to Digital Image Interpretation. Cell Mol Bioeng. 3:163-170.

Gardner, M.K., M. Zanic, and J. Howard. 2013. Microtubule catastrophe and rescue. Curr Opin Cell Biol. 25:14-22.

Gudimchuk, N.B., and J.R. McIntosh. 2021. Regulation of microtubule dynamics, mechanics and function through the growing tip. Nat Rev Mol Cell Biol. 
693

694

695

696

697

698

699

700

701

702

703

704

705

706

707

708

709

710

711

712

713

714

715

716

717

718

719

720

721

722

723

724

725

726

727

728

729

730

731

732

733

734

735

736

Howard, J., and A.A. Hyman. 2003. Dynamics and mechanics of the microtubule plus end. Nature. 422:753-758.

Kim, T., and L.M. Rice. 2019. Long-range, through-lattice coupling improves predictions of microtubule catastrophe. Mol Biol Cell. 30:1451-1462.

Leduc, C., F. Ruhnow, J. Howard, and S. Diez. 2007. Detection of fractional steps in cargo movement by the collective operation of kinesin-1 motors. Proc Natl Acad Sci U S A. 104:10847-10852.

Mahamdeh, M., and J. Howard. 2019. Implementation of Interference Reflection Microscopy for Label-free, High-speed Imaging of Microtubules. J Vis Exp.

Mahamdeh, M., S. Simmert, A. Luchniak, E. Schaffer, and J. Howard. 2018. Label-free highspeed wide-field imaging of single microtubules using interference reflection microscopy. J Microsc. 272:60-66.

Margolin, G., I.V. Gregoretti, T.M. Cickovski, C. Li, W. Shi, M.S. Alber, and H.V. Goodson. 2012. The mechanisms of microtubule catastrophe and rescue: implications from analysis of a dimer-scale computational model. Mol Biol Cell. 23:642-656.

Maurer, S.P., N.I. Cade, G. Bohner, N. Gustafsson, E. Boutant, and T. Surrey. 2014. EB1 accelerates two conformational transitions important for microtubule maturation and dynamics. Curr Biol. 24:372-384.

McIntosh, J.R., E. O'Toole, G. Morgan, J. Austin, E. Ulyanov, F. Ataullakhanov, and N. Gudimchuk. 2018. Microtubules grow by the addition of bent guanosine triphosphate tubulin to the tips of curved protofilaments. J Cell Biol. 217:2691-2708.

Mickolajczyk, K.J., A.S.I. Cook, J.P. Jevtha, J. Fricks, and W.O. Hancock. 2019a. Insights into Kinesin-1 Stepping from Simulations and Tracking of Gold Nanoparticle-Labeled Motors. Biophys J. 117:331-345.

Mickolajczyk, K.J., E.A. Geyer, T. Kim, L.M. Rice, and W.O. Hancock. 2019b. Direct observation of individual tubulin dimers binding to growing microtubules. Proc Natl Acad Sci U S A. 116:7314-7322.

Nogales, E., S.G. Wolf, and K.H. Downing. 1998. Structure of the alpha beta tubulin dimer by electron crystallography. Nature. 391:199-203.

Ortega-Arroyo, J., and P. Kukura. 2012. Interferometric scattering microscopy (iSCAT): new frontiers in ultrafast and ultrasensitive optical microscopy. Phys Chem Chem Phys. 14:15625-15636.

Piedra, F.A., T. Kim, E.S. Garza, E.A. Geyer, A. Burns, X. Ye, and L.M. Rice. 2016. GDP-to-GTP exchange on the microtubule end can contribute to the frequency of catastrophe. Mol Biol Cell. 27:3515-3525.

Prahl, L.S., B.T. Castle, M.K. Gardner, and D.J. Odde. 2014. Quantitative analysis of microtubule self-assembly kinetics and tip structure. Methods Enzymol. 540:35-52.

Prosser, S.L., and L. Pelletier. 2017. Mitotic spindle assembly in animal cells: a fine balancing act. Nat Rev Mol Cell Biol. 18:187-201.

Rice, L.M., E.A. Montabana, and D.A. Agard. 2008. The lattice as allosteric effector: structural studies of alphabeta- and gamma-tubulin clarify the role of GTP in microtubule assembly. Proc Natl Acad Sci U S A. 105:5378-5383.

Ruhnow, F., D. Zwicker, and S. Diez. 2011. Tracking single particles and elongated filaments with nanometer precision. Biophys J. 100:2820-2828. 
737 Schneider, C.A., W.S. Rasband, and K.W. Eliceiri. 2012. NIH Image to ImageJ: 25 years of image $738 \quad$ analysis. Nat Methods. 9:671-675.

739 Uppalapati, M., Y.-M. Huang, S. Shastry, T.N. Jackson, and W.O. Hancock. 2009. Microtubule 740 motors in microfluidics. Artech House, Boston, MA.

741 VanBuren, V., D.J. Odde, and L. Cassimeris. 2002. Estimates of lateral and longitudinal bond

$742 \quad$ energies within the microtubule lattice. Proc Natl Acad Sci U S A. 99:6035-6040.

743 Vemu, A., J. Atherton, J.O. Spector, C.A. Moores, and A. Roll-Mecak. 2017. Tubulin isoform

744 composition tunes microtubule dynamics. Mol Biol Cell. 28:3564-3572.

745 Young, G., and P. Kukura. 2019. Interferometric Scattering Microscopy. Annu Rev Phys Chem.

$746 \quad 70: 301-322$. 
A

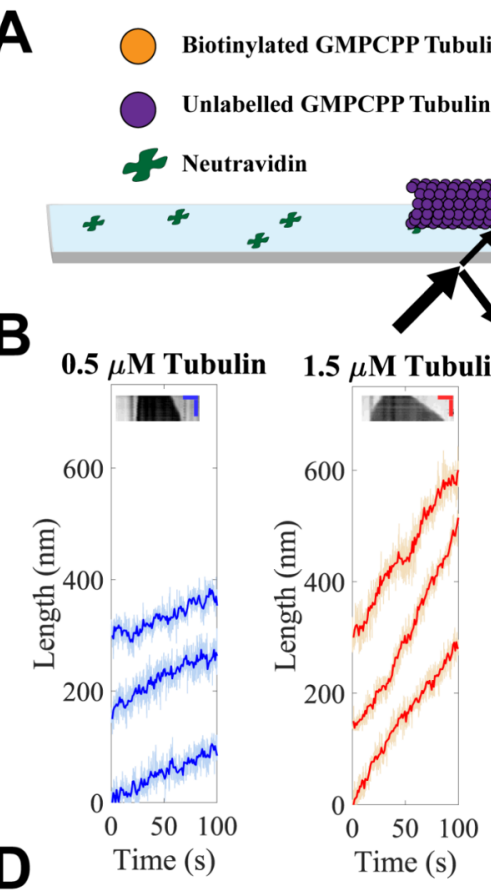

D

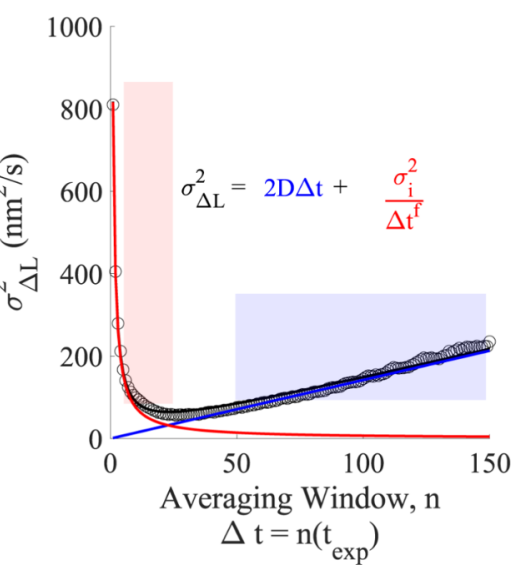

Figure 1
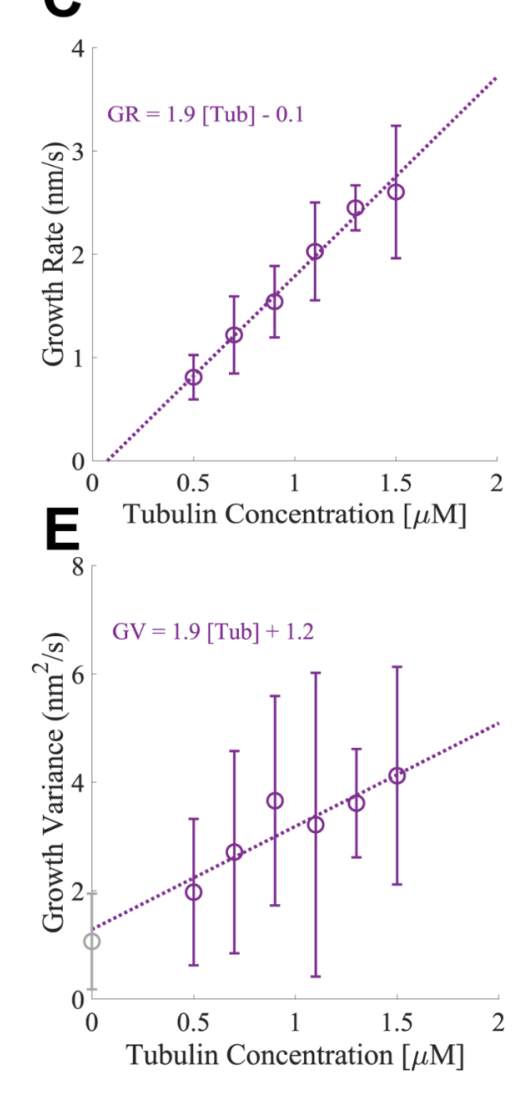
Figure 1. Microtubules growing in GMPCPP show slow growth and small fluctuations.

(A) Schematic of the dynamics assay. Biotinylated bovine GMPCPP microtubule seeds (orange) are attached to the cover slip through neutravidin, free unlabeled bovine tubulin is added to the flow cell in the presence of $1 \mathrm{mM} \mathrm{Mg-GMPCPP}$, and growing microtubules are visualized using Interference Reflection Microscopy (IRM) (image in inset; scale bar is $2 \mu \mathrm{m})$.

(B) Example traces of microtubule plus-end growth in the presence of GMPCPP at low (blue: $0.5 \mu \mathrm{M}$ ) and high (red: $1.5 \mu \mathrm{M}$ ) tubulin concentrations. Light traces show raw end positions at $10 \mathrm{fps}$, and darker lines represent a 10 frame $(1 \mathrm{~s})$ boxcar average. Kymographs are shown as insets above the traces. Vertical scale bar is 10 minutes and horizontal scale bar is $1 \mu \mathrm{m}$.

(C) Average growth rates versus free GMPCPP tubulin concentration. Error bars show the standard deviation of the growth rate at each concentration $(n=21,59,16,51,9$, and 23 microtubules). The dotted line shows the linear fit, weighted by the inverse of the standard error of the mean (1/SEM).

(D) Measurement of the growth variance for a single microtubule. Instantaneous displacements $(\Delta \mathrm{L})$ are recorded over different averaging windows, where $\mathrm{n}$ refers to the number of frames with exposure time $t_{\exp }$ of $0.1 \mathrm{~s}$ averaged, and the variance of $\Delta \mathrm{L}$ is plotted as a function of $\mathrm{n}$ (open black dots). The red line and shaded region show the fall in variance (approximately $1 / \Delta \mathrm{t}^{1}$ ) due to averaging out measurement error. The linear region denoted by the blue line and shading shows a crossover point where the measurement error becomes minimal and the increased variance is due to fluctuations around a mean growth rate. The black curve denotes the sum of these two phases. Reported values for growth variance in the paper refer to the ' $\mathrm{D}$ ' term in the linear regime.

(E) Average growth variance, resulting from fluctuations in microtubule growth, as a function of free tubulin. Error bars show the standard deviation of the measurement, and the linear fit is weighted by 1/SEM. A positive y-intercept is extrapolated back from the measurements at $1.2 \mathrm{~nm}^{2} / \mathrm{s}$, which matches closely to our experimental noise floor measured on stationary taxol-stabilized GMPCPP microtubule seeds (gray open circle), $0.97 \mathrm{~nm}^{2} / \mathrm{s}$. 


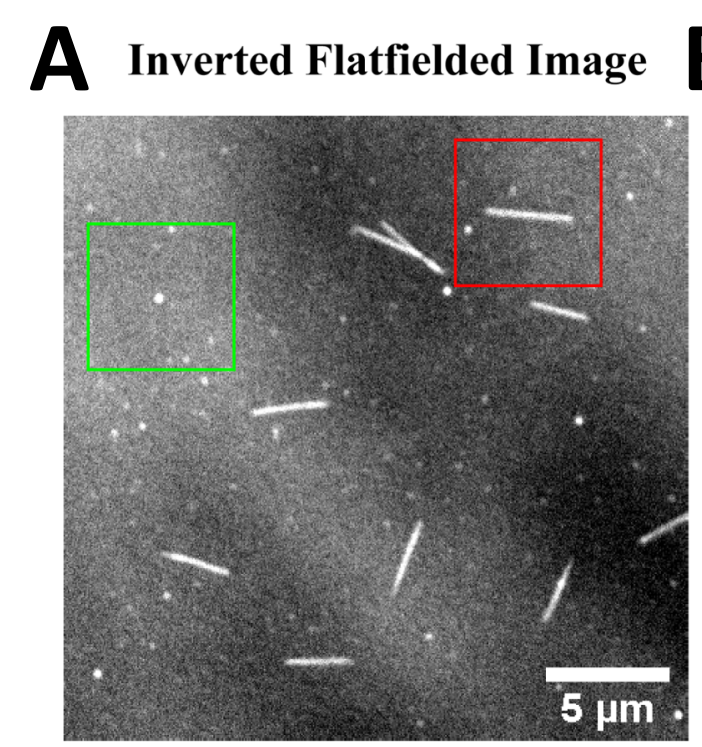

D

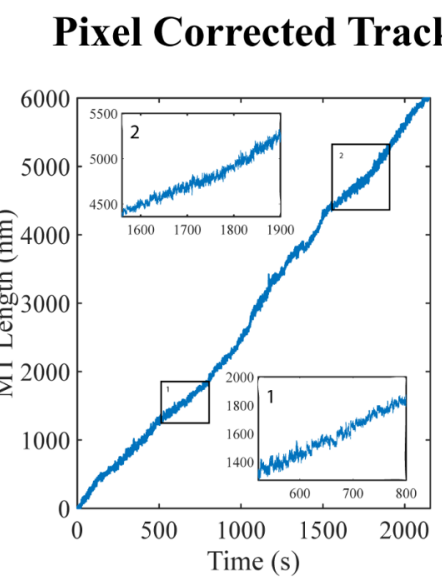

B Microtubule

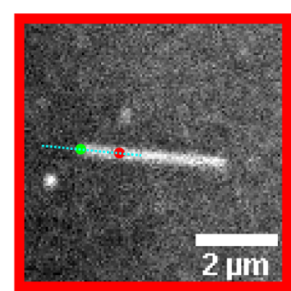

$6 \times 10^{4}$

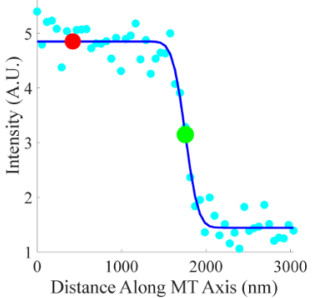

E

Sub-pixel Corrected

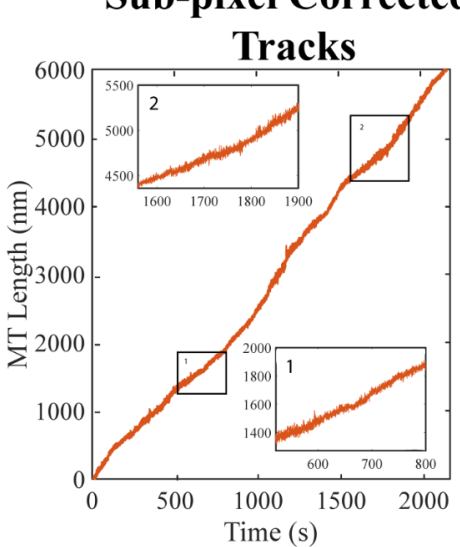

Figure 1 - figure supplement 1

C Fiduciary Marker

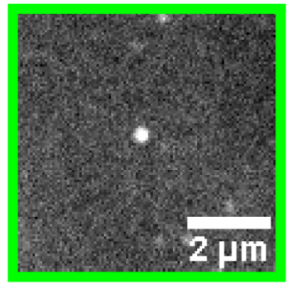

$\times 10^{4}$
8

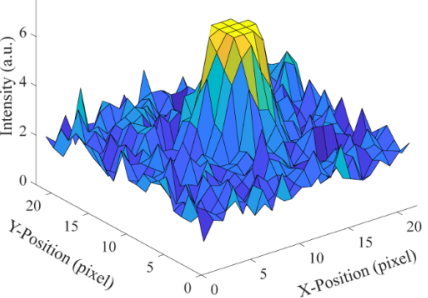

F

Mean Squared Displacement

of Concatenated Traces

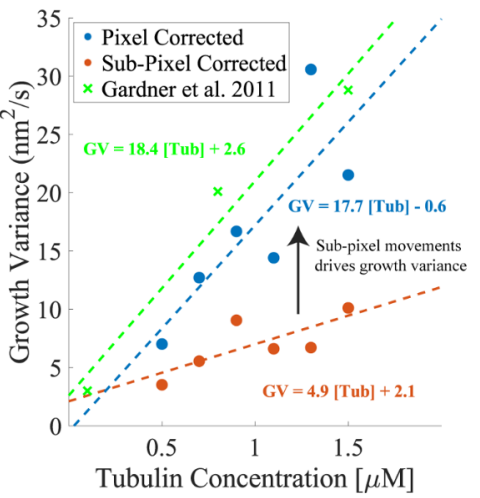


Figure 1 - figure supplement 1. Growth variance significantly decreases with sub-pixel correction of microtubule growth.

(A) Representative Interference Reflection Microscopy image of microtubules and fiduciary surface marks. Image is flat fielded to normalize for illumination heterogeneity, and inverted for visualization and analysis.

(B) Snapshot of a growing microtubule, showing the tip tracking algorithm (Demchouk et al., 2011; Gardner et al., 2011; Prahl et al., 2014). A line (cyan) is drawn along the microtubule backbone using user defined points (top), and the microtubule intensity along the backbone is plotted (bottom) and fit by a Gaussian survival function (dark blue line). A stationary reference point for microtubule length along the microtubule backbone is shown as a red dot in both panels, and the tip position, defined as the midpoint of the survival function, is shown by a green dot in both panels.

(C) Several fiduciary marks (top panel) are used in each video to correct for sub-pixel drift during image acquisition. The fiduciary marks are fit to a Gaussian profile (bottom panel) using FIESTA (Ruhnow et al., 2011), and microtubule positions are corrected using a custom drift correction MATLab script.

(D) Concatenated traces of 13 different microtubules growing at $1.5 \mu \mathrm{M}$ tubulin in GMPCPP, determined by the tracking algorithm developed in previous work (Demchouk et al., 2011; Gardner et al., 2011; Prahl et al., 2014). Microtubule growth was calculated as the distance between the backbone position shown (panel B, red point) and the tip position (panel B, green point). Insets 1 and 2 show small sub-pixel jumps during growth.

(E) The same concatenated growth trace as panel D, following sub-pixel drift correction using several fiduciary marks. Comparison of inset regions 1 and 2 demonstrates that failing to perform sub-pixel drift correction can inflate the estimates of the fluctuations.

(F) The resulting measurement of the growth variance using mean squared displacement (MSD) of the concatenated growth traces, as in (Gardner et al., 2011) (red points and line). Growth variance measured before sub-pixel registration (blue points and line) matched previous results, but when sub-pixel stage movements were subtracted (orange points and line), the growth variance decreased 4 -fold. 
Figure 1 - figure supplement 2
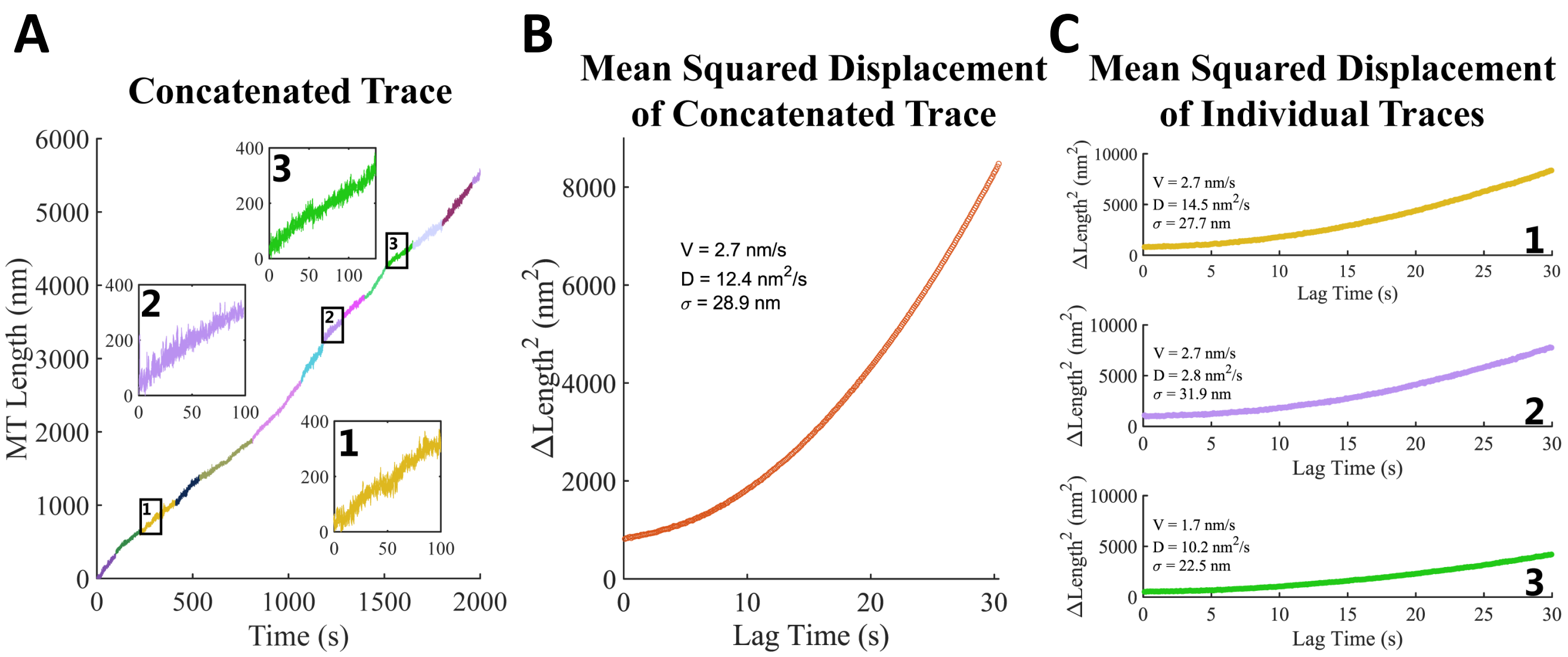
813 Figure 1 - figure supplement 2. Variations between microtubules growing under identical 814 conditions.

815 (A) Concatenated traces of 13 different microtubules growing at $1.5 \mu \mathrm{M}$ free GMPCPP 
Figure 1 - figure supplement 3
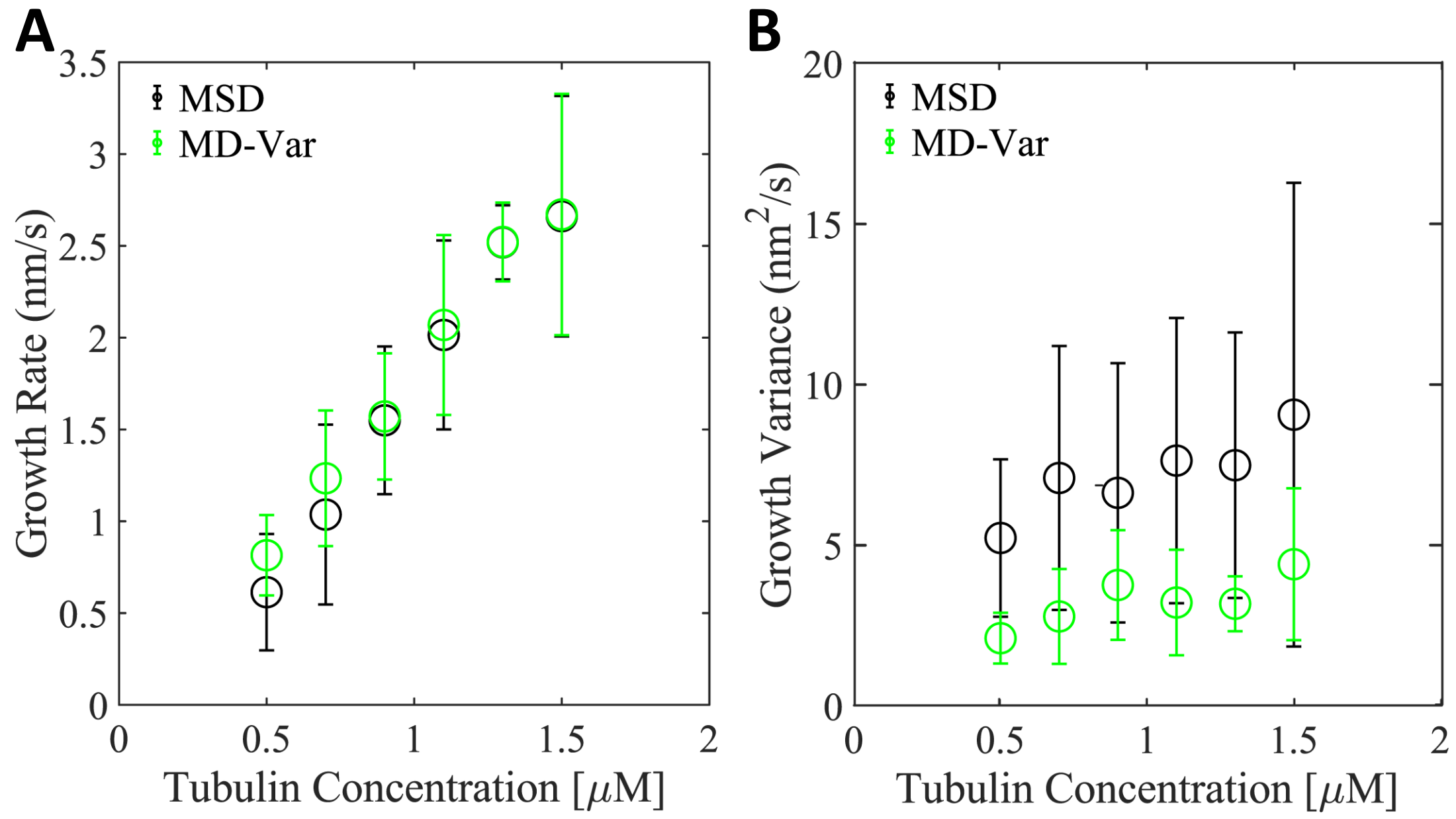
827 Figure 1 - figure supplement 3. MD-Var provides a better estimate for the growth variance 828 than MSD.

(A) Average growth rate as a function of free tubulin measured by Mean Squared Displacement (MSD - Black) or by Mean Displacement and Variance (MD-Var - Green); error bars show the standard deviation for the population of $\mathrm{n}=9$ to 59 microtubules. This comparison shows that the two approaches are in relative agreement for estimating growth rate.

(B) Average growth variance as a function of free tubulin measured by Mean Squared Displacement (MSD - Black) or by Mean Displacement and Variance (MD-Var - Green); error bars show the standard deviation of the population of $n=9$ to 59 microtubules. Note that both the mean and the range of growth variance values were larger for the MSD compared to the MD-Var analysis. 


\section{Figure 1 - figure supplement 4}
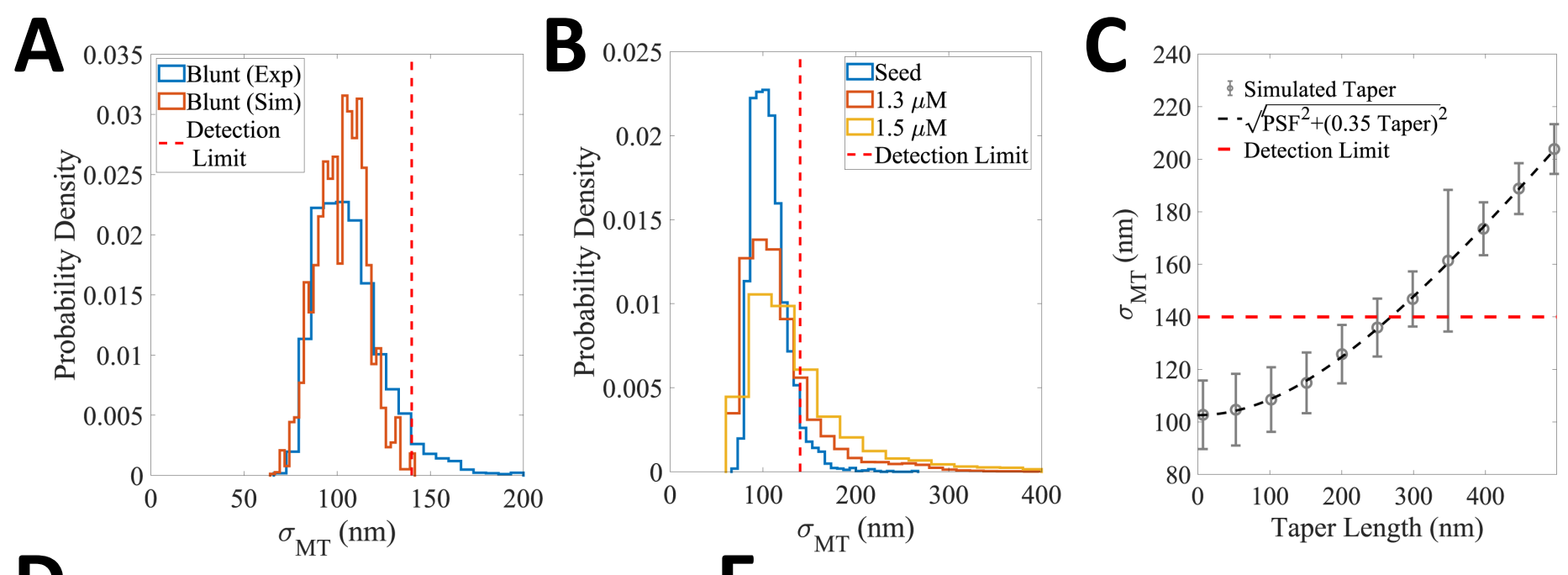

D

E

\begin{tabular}{c|c|c|c}
\hline Sample & $\begin{array}{c}\% \text { Over } \\
\text { Detection }\end{array}$ & $\begin{array}{c}\text { Mean } \\
\text { Taper* }\end{array}$ & $\begin{array}{c}\text { Max } \\
\text { Tapers ** }\end{array}$ \\
\hline Seed & $5 \%$ & $18 \mathrm{~nm}$ & $623 \mathrm{~nm}$ \\
\hline $1.3 \mu \mathrm{M}$ & $19 \%$ & $82 \mathrm{~nm}$ & $1192 \mathrm{~nm}$ \\
\hline $1.5 \mu \mathrm{M}$ & $34 \%$ & $165 \mathrm{~nm}$ & $1386 \mathrm{~nm}$
\end{tabular}

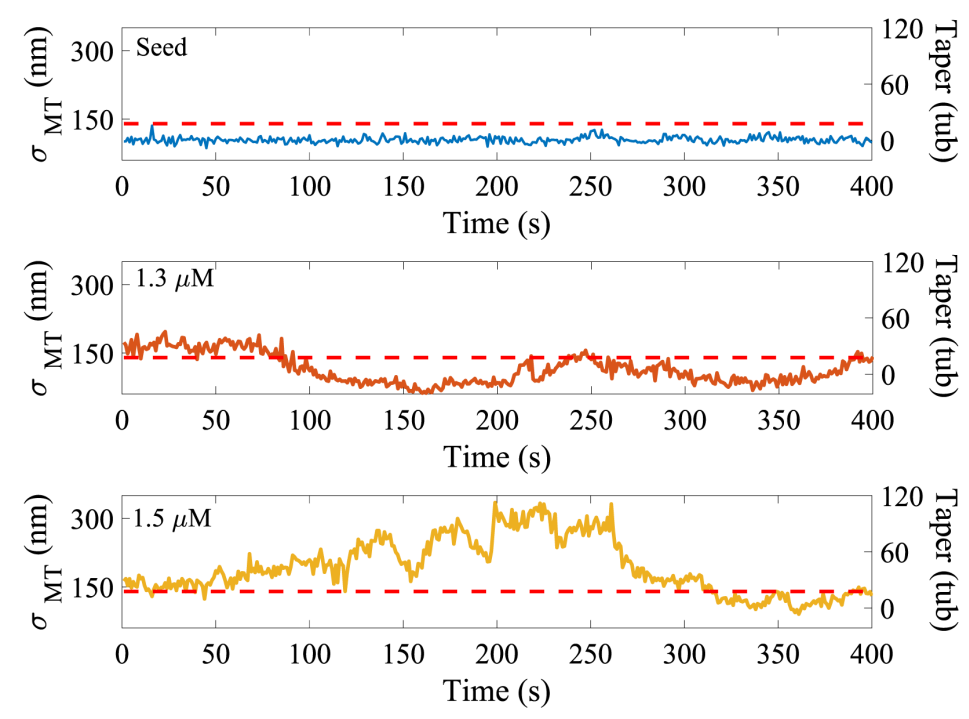


Figure 1 - figure supplement 4. Transient tapers fluctuate around a constant value and occur more often at higher free tubulin concentrations.

(A) Tip taper sizes were estimated from the standard deviation of the survival function used to fit the fall off of intensity at the tip $\left(\sigma_{M T}\right)$. To determine the minimum detectable taper, simulated images of blunt microtubules were generated by using model convolution based on our microscope setup and experimental measurement noise. The distribution of tip standard deviations from these simulated images is shown in orange. We defined the minimal experimentally detectable plus-tip taper as three times the standard deviation of this distribution (red dashed line at $140 \mathrm{~nm}$ ). The corresponding distribution from experiments imaging blunt, taxol-stabilized GMPCPP seeds is shown in blue. Note that a small population of our blunt microtubules had $\sigma_{\mathrm{MT}}$ above the threshold; we attribute these apparent tapers to experimental noise, such as out of focus images that are not captured in the model convolution.

(B) Comparison of experimentally measured tip standard deviations for taxol-stabilized GMPCPP seeds (blue), and microtubules growing in $1.3 \mu \mathrm{M}$ free tubulin (orange) and 1.5 $\mu \mathrm{M}$ free tubulin (yellow) in the presence of GMPCPP. The detection limit of $140 \mathrm{~nm}$ is shown as a dotted red line. As the free tubulin concentration increased, the fraction of tip estimations above the detection limit increased.

(C) Calibration curve relating tip standard deviation from the survival function fit, $\sigma_{\mathrm{MT}}$, to the actual taper length. Model convolution was used to generate simulated images of microtubules with increasing uniform taper structures (Bohner et al., 2016; Demchouk et al., 2011; Maurer et al., 2014; Prahl et al., 2014; Ruhnow et al., 2011) where the taper length is defined as the distance between the longest and the shortest protofilament. The mean and SD of $\sigma_{\mathrm{MT}}$ for each set of images is plotted as a function of the taper length used to generate that set of images (Bohner et al., 2016; Maurer et al., 2014; Prahl et al., 2014). A theoretical relationship between $\sigma_{\mathrm{MT}}$ and taper length is calculated by adding in quadrature the standard deviation of the protofilament length, $\sigma_{P F}$, and the spread of the optical point spread function (PSF), $\sigma_{\mathrm{PSF}}$.

$$
\sigma_{M T}=\sqrt{\sigma_{P F}^{2}+\sigma_{P S F}^{2}}
$$

The relationship between the standard deviation of the protofilament length, $\sigma_{P F}$, and taper should theoretically be $\frac{1}{\sqrt{12}}(0.29)$, but due to increases in tracking error that occur at longer taper lengths the relationship was fit empirically using $\sigma_{\mathrm{PF}}=0.35^{*}$ Taper (Demchouk et al., 2011; Maurer et al., 2014).

$$
\sigma_{M T}=\sqrt{(0.35 * \text { Taper })^{2}+\sigma_{P S F}^{2}}
$$

This calibration curve was used to estimate taper length from the experimental $\sigma_{\mathrm{MT}}$ data. The threshold for a measurable taper (red dashed line at $140 \mathrm{~nm}$ ) corresponds to a minimum detectable taper length of $265 \mathrm{~nm}$.

(D) Tip taper quantification on blunt microtubules and microtubules growing in GMPCPP. '\% Over Detection' is defined as the proportion of $\sigma_{\mathrm{MT}}$ measurements above the $140 \mathrm{~nm}$ detection limit defined in panel A. Note the increasing percent of measurable tips with increasing free tubulin; the 5\% for the seeds represents measurement error. * 'Mean Taper' is calculated by converting the $\sigma_{\mathrm{MT}}$ values for each condition to taper lengths using the calibration curve in Panel B, and taking the mean of this population. Note that these mean taper sizes are underestimates because any $\sigma_{\mathrm{MT}}$ below the detection limit was 
defined as having a taper of $0 \mathrm{~nm}$.** 'Max Tapers' is the average of the top $1 \%$ of tapers above the detection limit for each condition.

(E) Example time courses of the spread of the survival function ( $\sigma_{\mathrm{MT}}$, left y-axis) and the corresponding estimated taper length in tubulin subunits (right y-axis) for stabilized seeds (top), and microtubules growing in $1.3 \mu \mathrm{M}$ (middle) and $1.5 \mu \mathrm{M}$ (bottom) free tubulin in GMPCPP. The red dotted line is the measurement detection limit. Note that for the taxolstabilized GMPCPP seed (top panel-blue), the entire trace lies below the detection limit. The $1.3 \mu \mathrm{M}$ free tubulin trace (middle panel - orange) shows fluctuations of the taper length occurring over $10 \mathrm{~s}$ of seconds. The $1.5 \mu \mathrm{M}$ free tubulin trace (bottom panel yellow ) shows time-dependent fluctuations of taper size with a sustained period above the detection limit. 
bioRxiv preprint doi: https://doi.org/10.1101/2021.11.24.469935; this version posted November 25, 2021. The copyright holder for this preprint (which was not certified by peer review) is the author/funder, who has granted bioRxiv a license to display the preprint in perpetuity. It is made available under aCC-BY 4.0 International license.

A

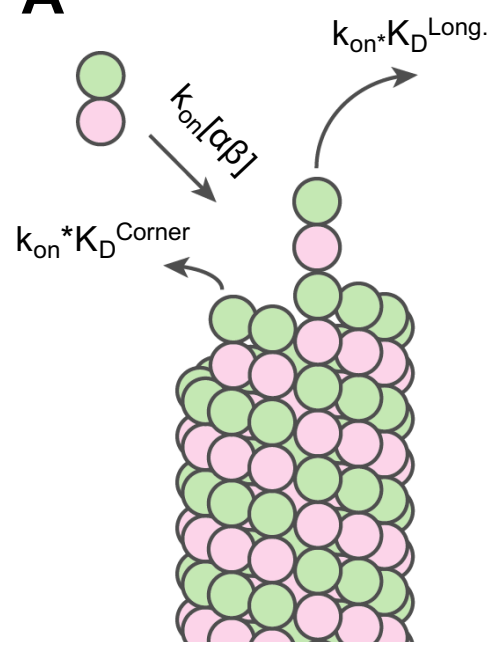

B

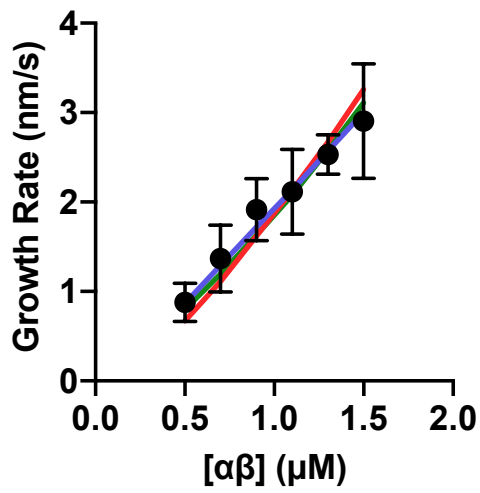

Figure 2

D

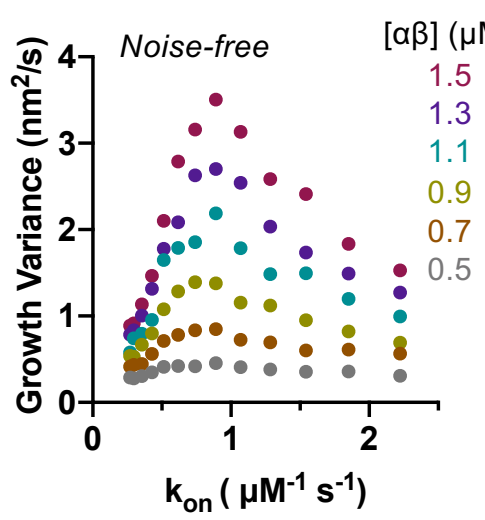

E

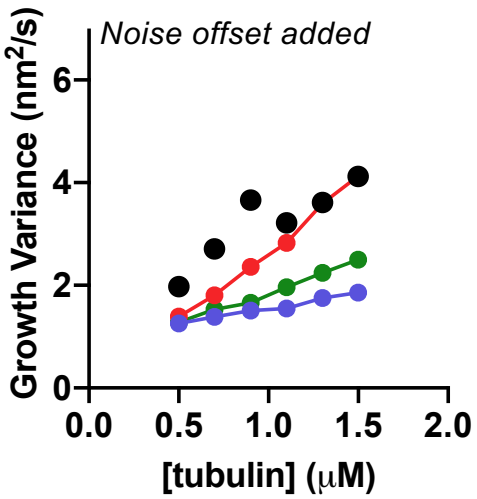

\begin{tabular}{r|c|c|c}
$\mathbf{k}_{\text {on }}\left(\boldsymbol{\mu} \mathbf{M}^{-1} \mathbf{s}^{-1}\right)$ & $\mathbf{0 . 2 7}$ & .74 & 2.2 \\
\hline $\mathrm{K}_{\mathrm{D}}^{\text {Long. }}$ & $1.0 \mu \mathrm{M}$ & $104 \mu \mathrm{M}$ & $14 \mathrm{mM}$ \\
\hline $\mathrm{K}_{\mathrm{D}}$ Corner & $25 \mathrm{nM}$ & $25 \mathrm{nM}$ & $25 \mathrm{nM}$
\end{tabular}

C

$F$

Best model from fitting to growth rates and fluctuations
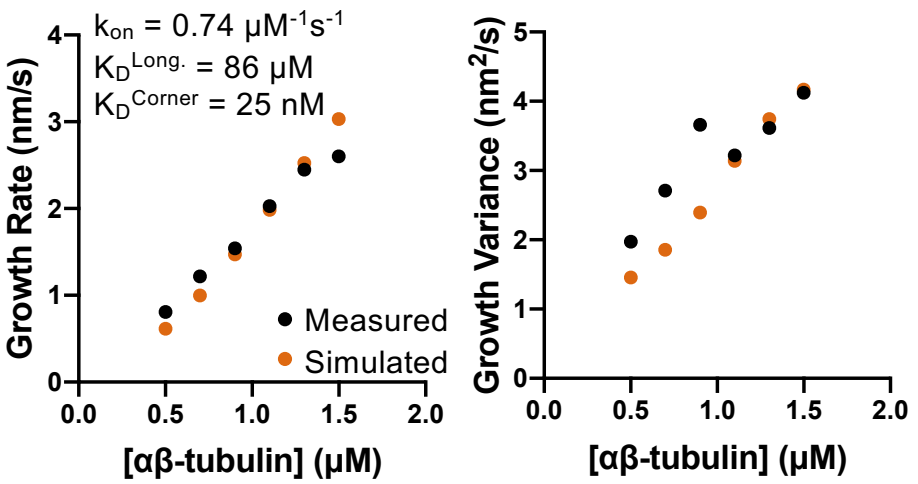
Figure 2. Simulations recapitulate GMPCPP growth rates for a wide range of association rates and longitudinal affinity, but fluctuations are only captured in a narrower range.

(A) Cartoon showing the dominant factors that determine the concentration-dependent growth rates: association rate is determined as $\mathrm{k}_{o n}{ }^{*}[\alpha \beta]$, where $\mathrm{k}_{\mathrm{on}}$ is the on-rate constant, and dissociation rates from longitudinal (no lateral neighbors) and corner (one lateral neighbor) sites are determined as $\mathrm{k}_{\mathrm{on}} * \mathrm{~K}_{\mathrm{D}}$, where $\mathrm{K}_{\mathrm{D}}$ represents the dissociation constant for the given type of site (see Methods). the model to the measurements for three different assumed $\mathrm{k}_{\text {on }}$ values (blue: $0.27 \mu \mathrm{M}^{-1} \mathrm{~s}^{-1}$, red: $0.74 \mu \mathrm{M}^{-1} \mathrm{~s}^{-1}$, green: $\left.2.22 \mu \mathrm{M}^{-1} \mathrm{~s}^{-1}\right)$.

(C) Fitted parameters for the three fits depicted in B.

(D) Fluctuations around the average simulated growth rate as produced by fitting the model to the measurements for a larger set of assumed $\mathrm{k}_{\text {on }}$ values than in $\mathrm{B}$. The magnitude of fluctuations in simulations shows a bell-shaped dependence on $\mathrm{k}_{\mathrm{on}}$, reaching the highest values in the middle of the range explored.

(E) Measured (filled black circles; data from Fig. 1) and simulated (filled circles connected by lines; color coding as in B) fluctuations around the average growth rate. A 'noise offset' of $0.97 \mathrm{~nm}^{2} \mathrm{~s}^{-1}$ (see Fig. 1E) has been added to the simulation data to facilitate comparison with the measurements.

921

(F) Result of fitting the model to growth rates (left) and fluctuations (right) simultaneously. Measured values are from Fig. 1 and depicted as filled circles; values from simulations are $\mathrm{k}_{\text {on }}: 0.74 \mu \mathrm{M}^{-1} \mathrm{~s}^{-1}, \mathrm{~K}_{\mathrm{D}, \text { long }}: 86 \mu \mathrm{M}, \mathrm{K}_{\mathrm{D} \text {, corner }}: 25 \mathrm{nM}$. 

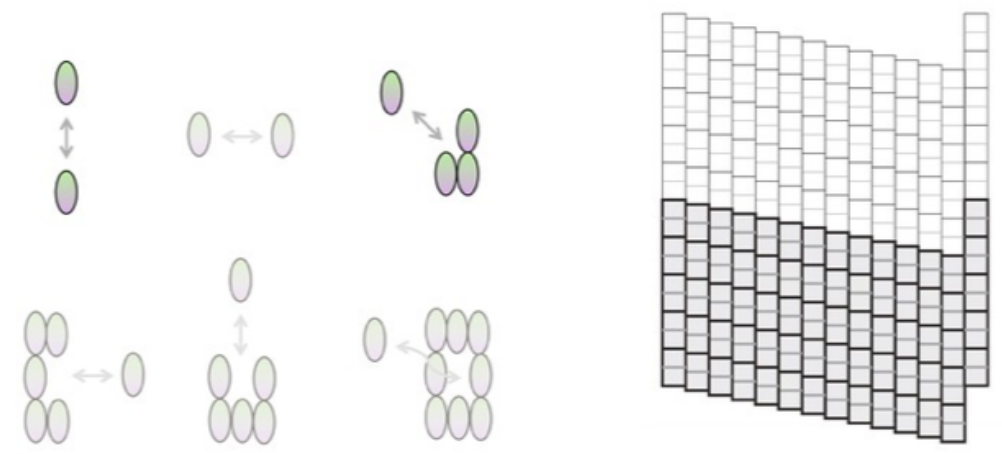

\author{
Initial conditions: \\ + template 'seed' \\ +13 possible association events
}

Then simulate MT dynamics one biochemical event at a time. The relative probability of different events are determined from the characteristic rates or rate constants

\title{
C
}

Simulation algorithm:

0 . Start at time $\mathrm{T}=0 \mathrm{~s}$

1. determine 'execution time' for possible events.

2. Implement the event with the shortest execution time $\Delta T_{\text {fastest }}$

$=$ Advance simulation time: $T \rightarrow T+\Delta T_{\text {fastest }}$

$=$ Add new events and remove old events in response to the event that just occurred

\section{GOTO 1}

D

\section{$\mathrm{T}=0 \mathrm{~s}$}

13 associations

rate $k_{o n}[\alpha \beta]$

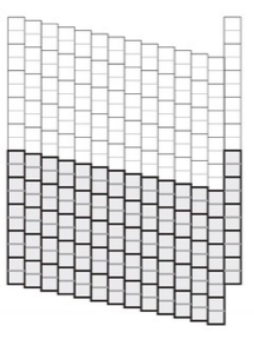

$\mathrm{T}=\Delta \mathrm{T}_{1} \mathrm{~s}$

13 associations rate $\mathrm{k}_{\mathrm{on}}[\alpha \beta]$

1 dissociation rate $\mathrm{k}_{\mathrm{on}}{ }^{*} \mathrm{~K}_{\mathrm{d}}$ Long

1 GTPase rate $\mathrm{k}_{\mathrm{GTP}}$ ase

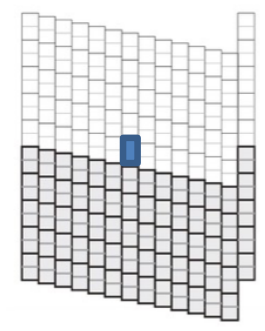

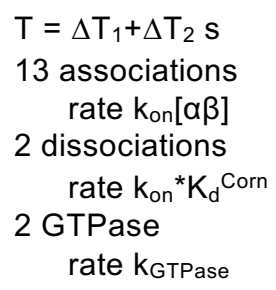

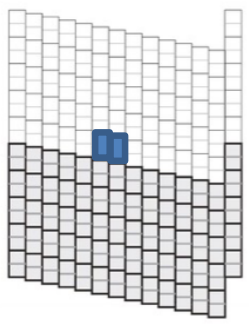

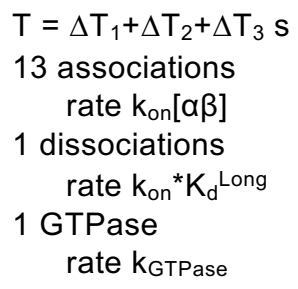
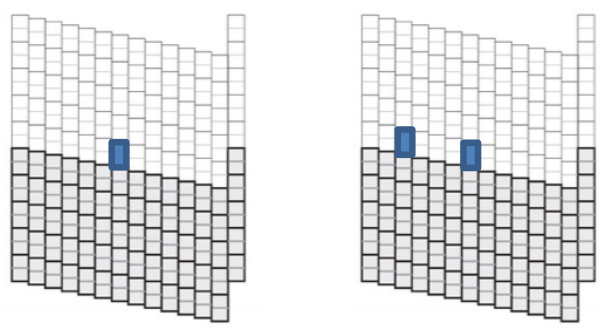

$\mathbf{E}$

Results of the simulation:

$+\mathrm{N}_{\alpha \beta}$ (number of tubulins in the microtubule) as a function of time

+ biochemical state (GTP/GDP) and environment (neighbors) for every tubulin

Comparing to experimental data:

+ Convert to length units to obtain growth rate

+ Analyze simulated growth rates and fluctuations as for experiments

+ Adjust model parameters to obtain best agreement between simulation and experiment 


\section{Figure 2 - figure supplement 1. Explanation and illustration of the model.}

(A) Cartoons illustrating binding modes for $\alpha \beta$-tubulin (shaded oval) that differ in the number of lateral and longitudinal contacts (neighbors). The binding modes (longitudinal, top left; corner, top right) that dominate elongation rates in our model are shown in bold colors.

(B) Representation of the microtubule lattice as a two-dimensional grid with a 'wraparound' boundary condition to model the seam. The darker rectangles represent the 'seed' that is used to initiate simulations. Accompanying text explains the initial conditions for starting simulations.

(C) Essence of the simulation algorithm. 'Execution times' are computed for each possible event (association, dissociation, GTP hydrolysis), and the event with the shortest execution time is implemented, accumulated simulation time incremented, and list of possible events updated. In this way, the individual events are chosen in a way that reflects their relative probability, reflected in their different characteristic rates.

(D) Explanatory cartoon illustrating the first few steps of a hypothetical simulation.

(E) Simulations output the number of tubulins in the microtubule as a function of time and, if desired, the location and biochemical state of tubulins within a user-specified distance of the microtubule end. The length vs time data can be analyzed for growth rate and fluctuations as for experimental measurements, and model parameters can be optimized to achieve the best fit to the desired experimental measurements. 
bioRxiv preprint doi: https://doi.org/10.1101/2021.11.24.469935; this version posted November 25, 2021. The copyright holder for this preprint (which was not certified by peer review) is the author/funder, who has granted bioRxiv a license to display the preprint in perpetuity. It is made available under aCC-BY 4.0 International license.

Figure 2 - Figure Supplement 2

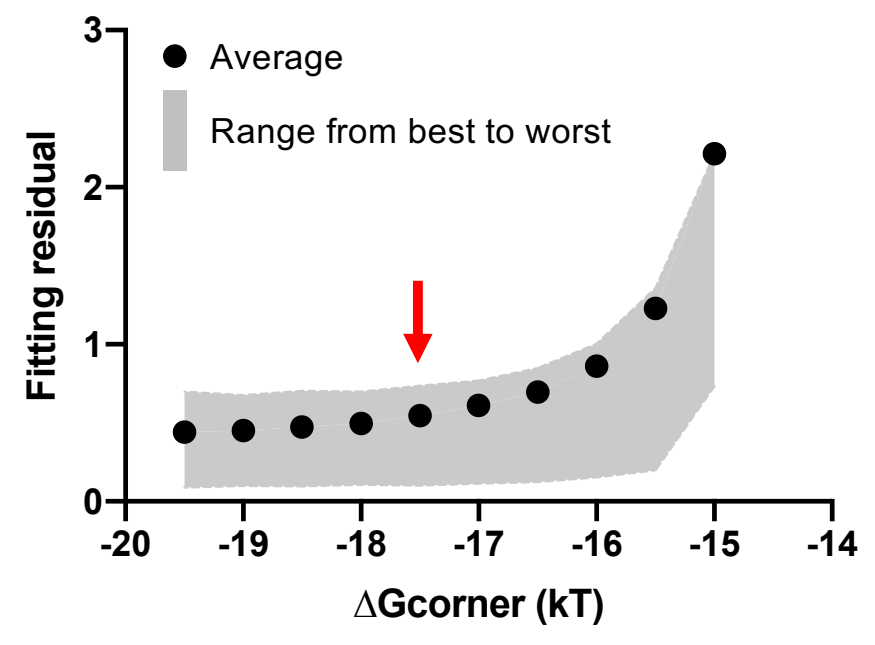




\section{Figure 2 - figure supplement 2. Model fit to measured growth rates as a function of corner}

946 The plot summarizes the result of fitting the model to the measured GMPCPP growth rates using

947 a variety of corner affinities. The x-axis range from -20 to $-14 \mathrm{kT}$ corresponds to dissociation

948 constants of $2 \mathrm{nM}$ to $0.83 \mu \mathrm{M}$. For each corner affinity, the model was fit to experimental growth

949 rates using a range of assumed $\mathrm{k}_{\text {on }}$ values (see Methods). Black circles represent the average

950 goodness of fit taken over all $\mathrm{k}_{\text {on }}$ values, and the boundaries of the grey shaded region show the

951 best and worst fit obtained from an individual $\mathrm{k}_{\text {on. }}$. As long as the corner affinity is sufficiently

952 strong, the model can fit the observed growth rates. The red arrow indicates the corner affinity (-

$95317.5 \mathrm{kT}$, corresponding to $25 \mathrm{nM}$ dissociation constant) used for Fig. 2F. 

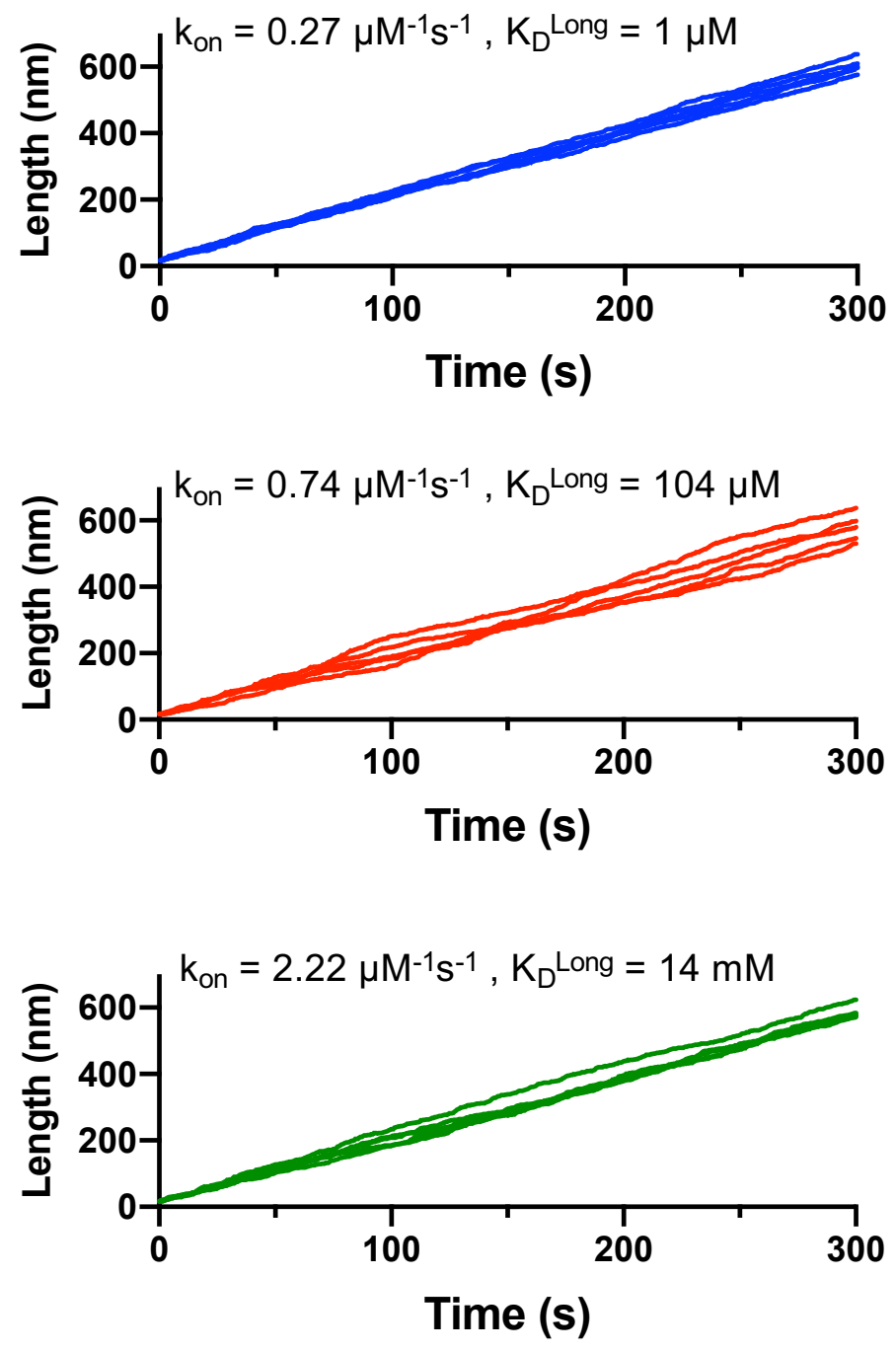
956 Figure 2 - figure supplement 3. Differing magnitude variation in simulated microtubule 957 growth at different association rate constants.

958 Plots of five traces of microtubule length vs time from independent simulations are shown for 959 three different choices of $\mathrm{k}_{\text {on }}\left(0.27,0.74,2.22 \mu \mathrm{M}^{-1} \mathrm{~s}^{-1}\right)$. The different degrees of divergence in 960 the length vs time traces for different regimes of association rate (with their correspondingly 961 different longitudinal affinities, see insets) reflect different magnitude variation in growth rates. 
bioRxiv preprint doi: https://doi.org/10.1101/2021.11.24 469935; this version posted November 25, 2021. The copyright holder for this preprint (which was not certified by peer review) is the author/funder, who has granted bioRxiv a license to display the preprint in perpetuity. It is made available under aCC-BY 4.0 International license.

Figure 2 - Figure Supplement 4

$$
\begin{aligned}
& \mathrm{K}_{\mathrm{on}}=.27 \mu \mathrm{M}^{-1} \mathrm{~s}^{-1} \\
& \mathrm{~K}_{\mathrm{D}} \text { Long }=1 \mu \mathrm{M}
\end{aligned}
$$

$\mathrm{k}_{\text {on }}=.74 \mu \mathrm{M}^{-1} \mathrm{~s}^{-1}$

$\mathrm{K}_{\mathrm{D}}$ Long $=104 \mu \mathrm{M}$

$$
\begin{aligned}
& \mathrm{K}_{\mathrm{on}}=2.22 \mu \mathrm{M}^{-1} \mathrm{~s}^{-1} \\
& \mathrm{~K}_{\mathrm{D}} \text { Long }=14.2 \mathrm{mM}
\end{aligned}
$$
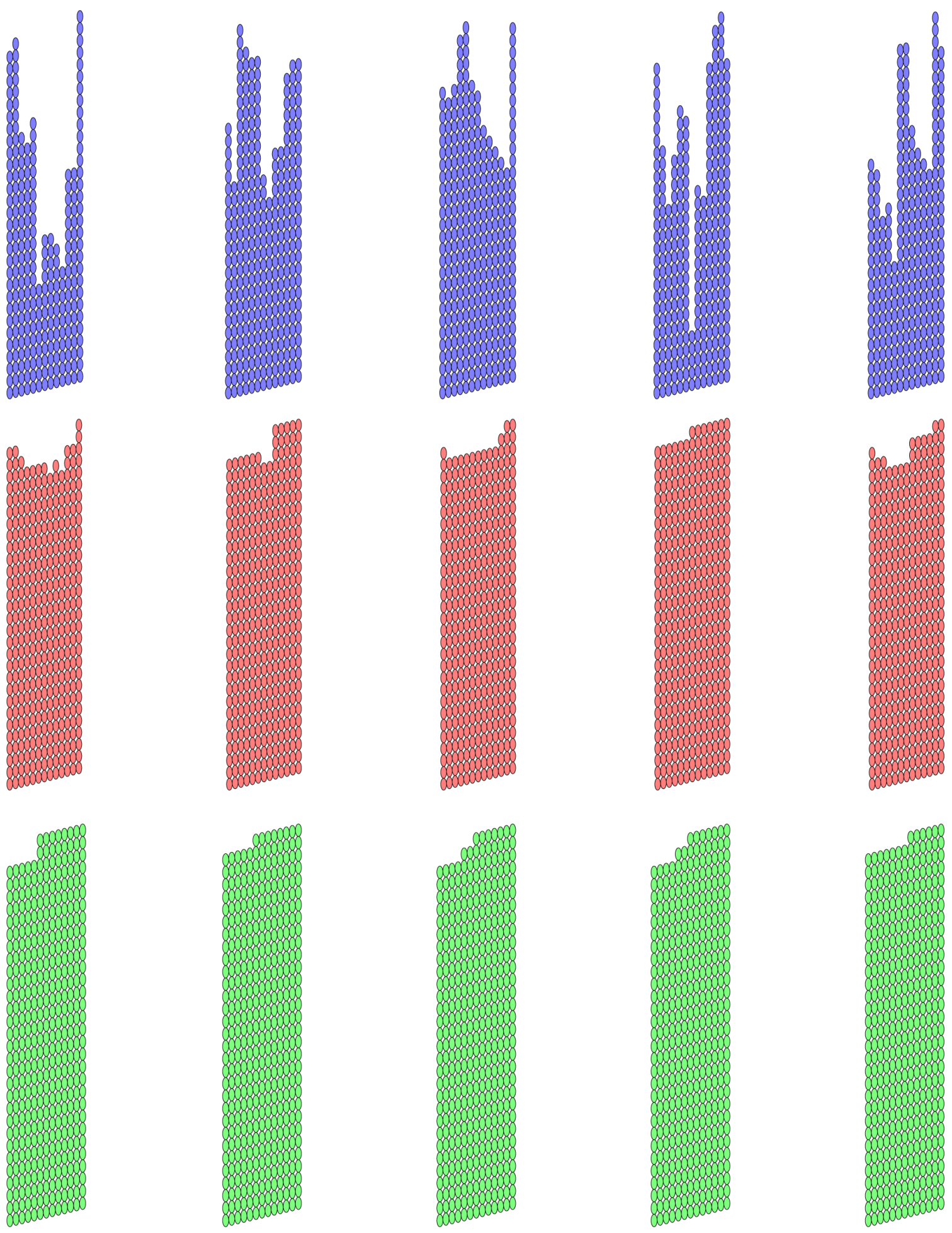
964 Figure 2 - figure supplement 4. The taper and roughness of microtubule end structures 965 from simulations depends on the regime of association rate and longitudinal affinity.

966 Representative microtubule end configurations from 5 independent simulations using three 967 different choices of $\mathrm{k}_{\text {on }}\left(0.27,0.74,2.22 \mu \mathrm{M}^{-1} \mathrm{~s}^{-1}\right)$. Tubulin subunits are represented as ovals, and 968 the microtubule lattice is represented as a two-dimensional grid with protofilaments 1 and 13 at 969 either extreme. Conditions of relatively slow association rate and strong longitudinal affinity (top 970 row) give rise to substantial taper (difference in length of the protofilaments) and these

971 microtubules can have multiple growth fronts. Conditions of relative fast association rate and

972 weak longitudinal affinity (bottom row) yield blunter microtubule ends and intermediate

973 conditions (middle row) yield slightly tapered ends with a few available growth fronts. 
bioRxiv preprint doi: https://doi.org/10.1101/2021.11.24.469935; this version posted November 25, 2021. The copyright holder for this preprint (which was not certified by peer review) is the author/funder, who has granted bioRxiv a license to display the preprint in perpetuity. It is made available under aCC-BY 4.0 International license.

Figure 2 - Figure Supplement 5
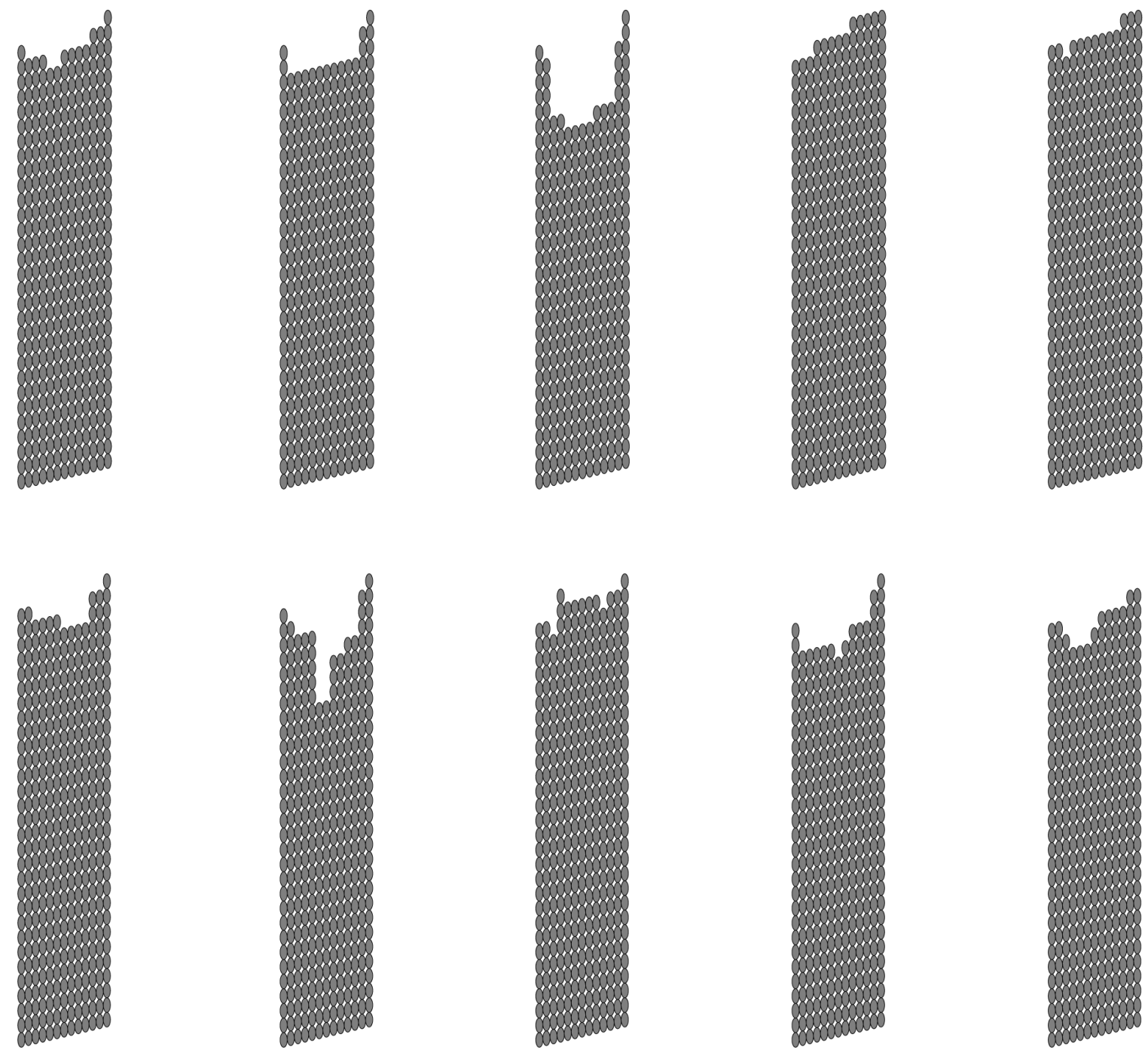
976 Figure 2 - figure supplement 5. Gallery of microtubule end tapers obtained using the best 977 fit model parameters.

978 Growth parameters used in Fig. $2 \mathrm{~F}\left(\mathrm{k}_{\mathrm{on}}: 0.74 \mu \mathrm{M}^{-1} \mathrm{~s}^{-1}, \mathrm{~K}_{\mathrm{D} \text {,long }}: 86 \mu \mathrm{M}, \mathrm{K}_{\mathrm{D} \text {,corner }}: 25 \mathrm{nM}\right.$.) were 979 obtained by fitting the model to both growth rates and fluctuations. Representative microtubule 980 end configurations from 10 independent simulations are shown. Tubulin subunits are represented 981 as ovals, and the microtubule lattice is represented as a two-dimensional grid with protofilaments 9821 and 13 at either extreme. End configurations here varied from more blunt ends to longer tapers 983 that are several tubulin subunits long.

984

985 

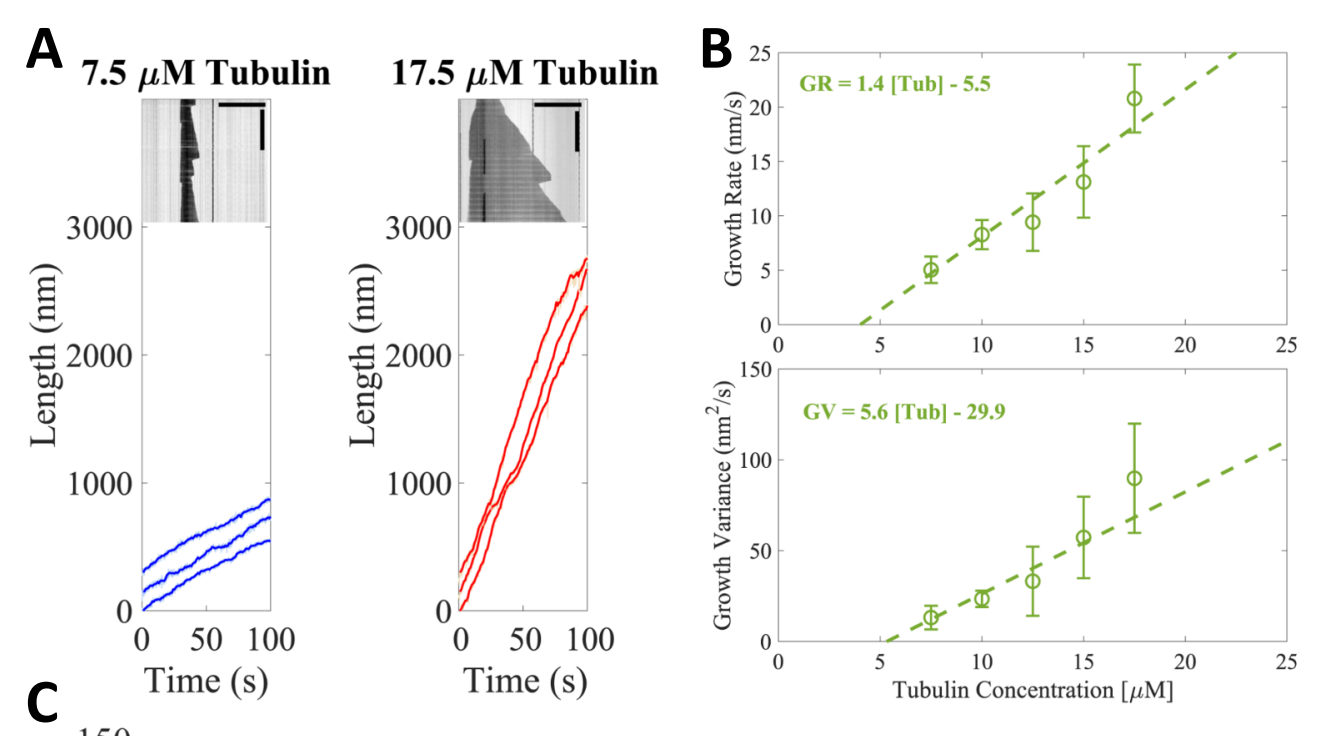

Figure 3

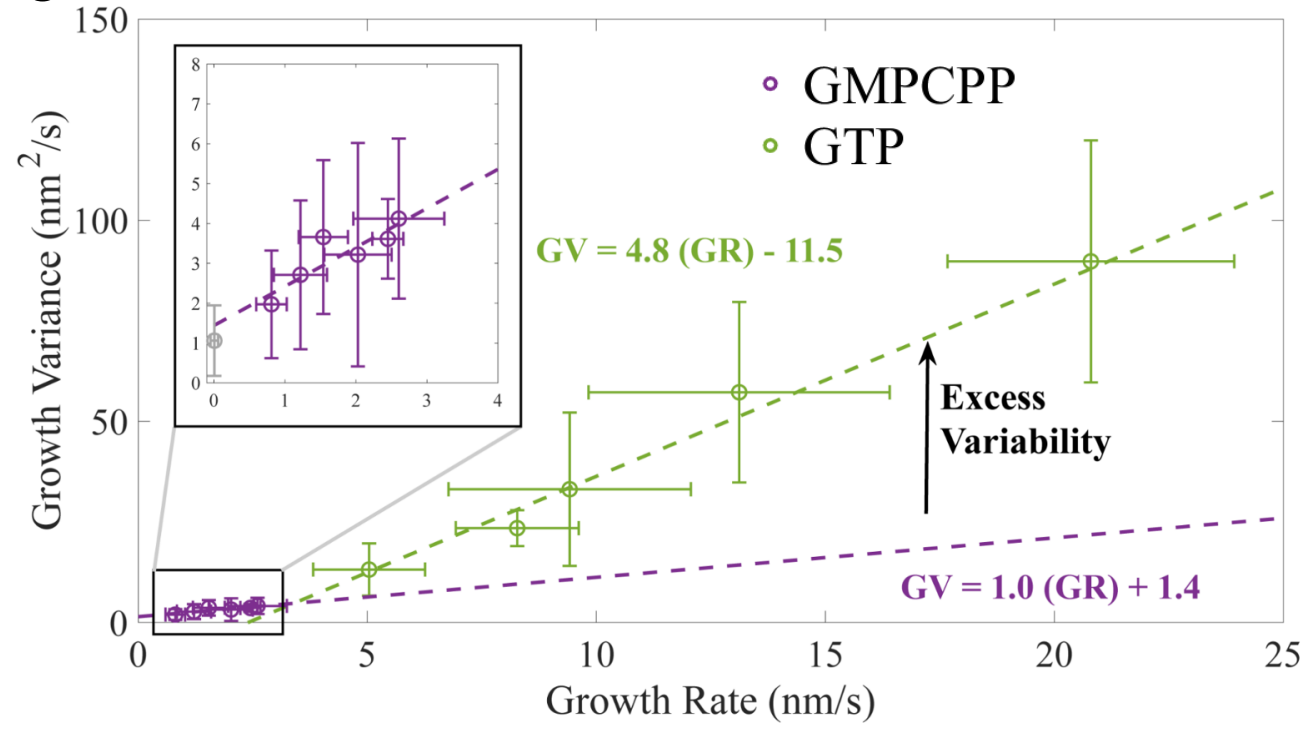


986 Figure 3. Microtubules growing in GTP show excess fluctuations compared to growth in 987 GMPCPP.

(A) Example tracks of microtubule growth in GTP at $7.5 \mu \mathrm{M}$ free tubulin (left, blue) and 17.5 $\mu \mathrm{M}$ free tubulin (right, red). The light shading represents the tip traces acquired at 10 frames per second and the darker traces denote a 10-frame boxcar average of the traces over time. Example kymographs of dynamic microtubules are shown as insets; horizontal scale bar is $10 \mu \mathrm{m}$ and the vertical scale bar is 5 minutes.

(B) Average microtubule growth rate (top) and average growth variance (bottom) as a $34,20,16,15,13$ growth events). A linear fit, weighted by 1/SEM, is shown as the dashed green line.

1000

1001

1002

1003

(C) Growth variance versus growth rate for microtubules grown in GMPCPP (purple) and GTP (green). Error bars are SD. The inset more clearly shows the linear relationship between growth rate and growth variance in GMPCPP (purple). The experimental noise floor is shown as the gray point in the inset. Dashed lines denote linear fits weighted by $1 / \mathrm{SEM}$ of the growth variance. 
Figure 3 - figure supplement 1
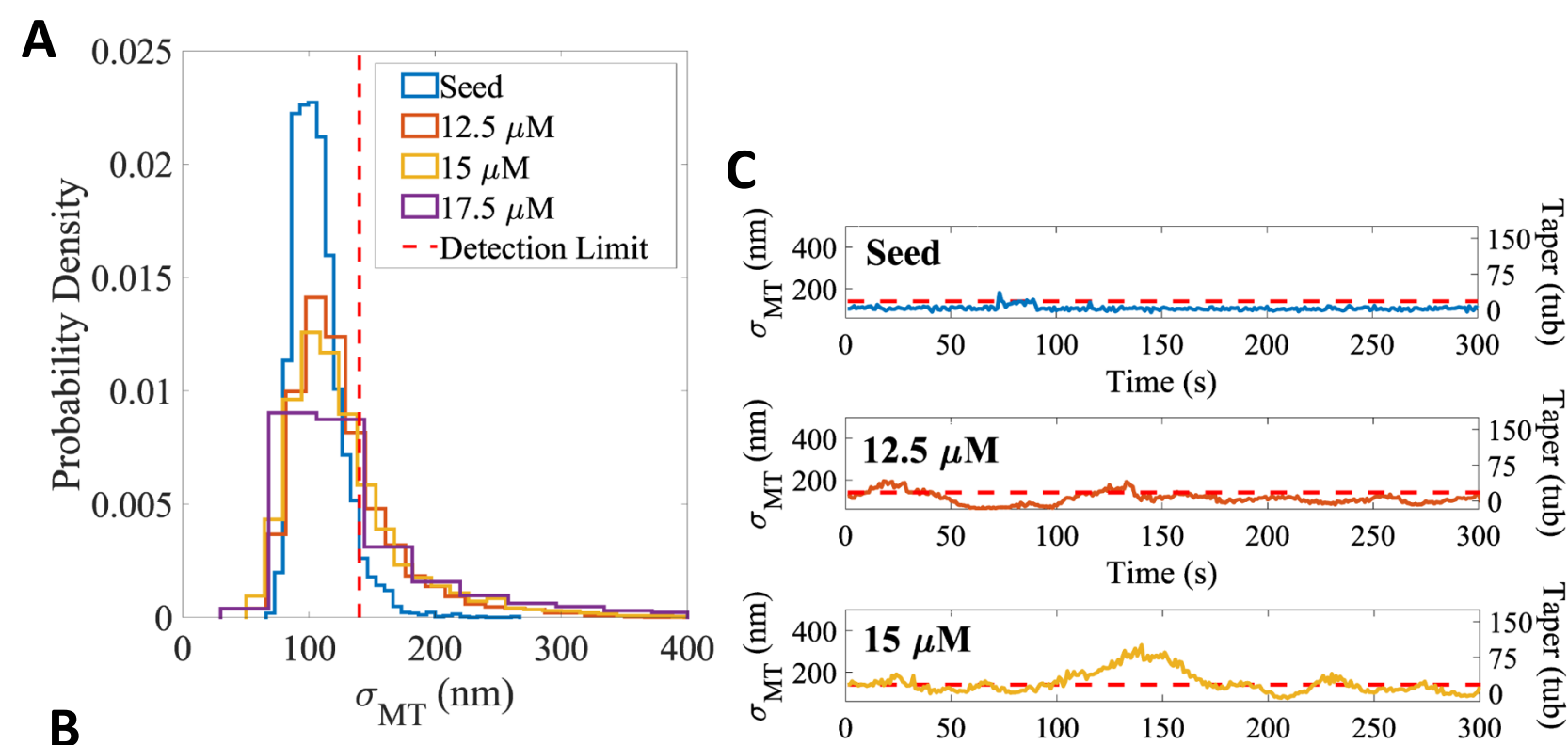

\begin{tabular}{c|c|c|c} 
Sample & $\begin{array}{c}\text { \% Over } \\
\text { Detection }\end{array}$ & $\begin{array}{c}\text { Mean } \\
\text { Taper* }\end{array}$ & $\begin{array}{c}\text { Max } \\
\text { Tapers ** }\end{array}$ \\
\hline Seed & $5 \%$ & $18 \mathrm{~nm}$ & $623 \mathrm{~nm}$ \\
\hline $12.5 \mu \mathrm{M}$ & $26 \%$ & $110 \mathrm{~nm}$ & $1091 \mathrm{~nm}$ \\
\hline $15 \mu \mathrm{M}$ & $28 \%$ & $124 \mathrm{~nm}$ & $1133 \mathrm{~nm}$ \\
\hline $17.5 \mu \mathrm{M}$ & $33 \%$ & $204 \mathrm{~nm}$ & $2628 \mathrm{~nm}$
\end{tabular}


1004 Figure 3 - figure supplement 1. Long transient tapers occur during microtubule growth in 1005 GTP.

1006

1007

1008

1009

1010

1011

1012

1013

1014

1015

1016

1017

1018

1019

1020

1021

1022

1023

1024

1025

1026

1027

1028
(A) Distribution of measured taper sizes $\left(\sigma_{\mathrm{MT}}\right)$ of microtubules growing in GTP. Red dotted line denotes the detection limit of $140 \mathrm{~nm}$. Note that as the free tubulin concentration increased, the proportion of measurements above the detection limit increased. Corresponding distributions for microtubules growing in GMPCPP are shown in Figure 1 - figure supplement 4B.

(B) Tip taper quantification for microtubules growing in GTP. '\% Over Detection' is defined as the proportion of $\sigma_{\mathrm{MT}}$ measurements above the $140 \mathrm{~nm}$ detection limit. Note the increasing percent of measurable tips with increasing free tubulin; the $5 \%$ for the seeds represents measurement error. * 'Mean Taper' is calculated by converting the $\sigma_{\mathrm{MT}}$ values for each condition to taper lengths using the calibration curve in Figure1-figure supplement $4 \mathrm{C}$, and taking the mean of this population. Note that these mean taper sizes are underestimates because any $\sigma_{\mathrm{MT}}$ below the detection limit was defined as having a taper of $0 \mathrm{~nm}$. ** 'Max Tapers' is the average of the top 1\% of tapers above the detection limit for each condition. Corresponding table for microtubules growing in GMPCPP is shown in Figure 1 - figure supplement 4D.

(C) Example time courses of the spread of the survival function ( $\sigma_{\mathrm{MT}}$, left y-axis) and the corresponding taper length in tubulin subunits (right y-axis) for stabilized seeds (top), and microtubules growing in increasing free tubulin concentrations in GTP. Red dotted line denotes the measurement detection limit of $140 \mathrm{~nm}$. Note that episodes where the taper length exceeds the detection limit increase in both duration and magnitude with increasing tubulin. 


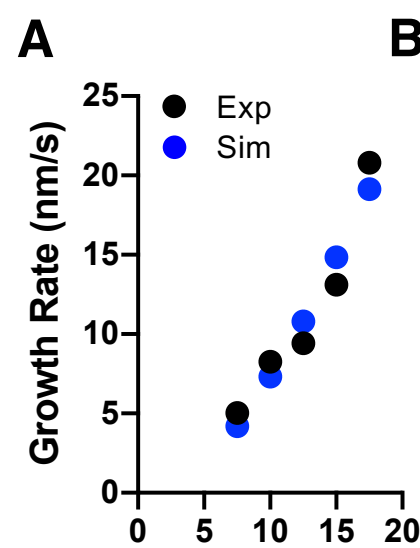

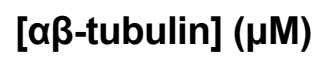

B

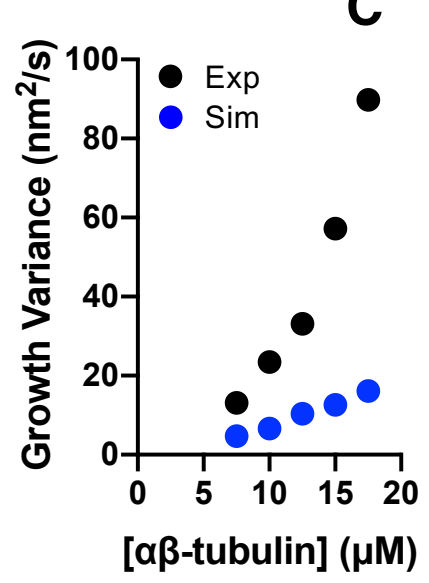

C

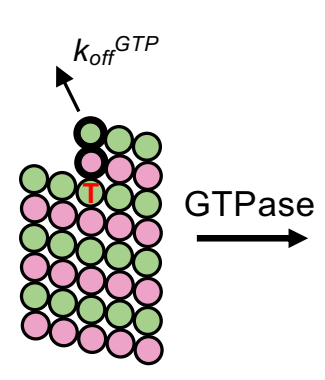

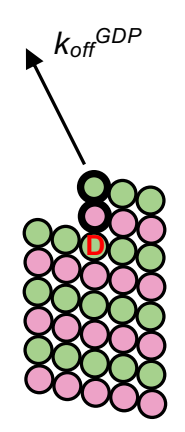

D

Figure 4
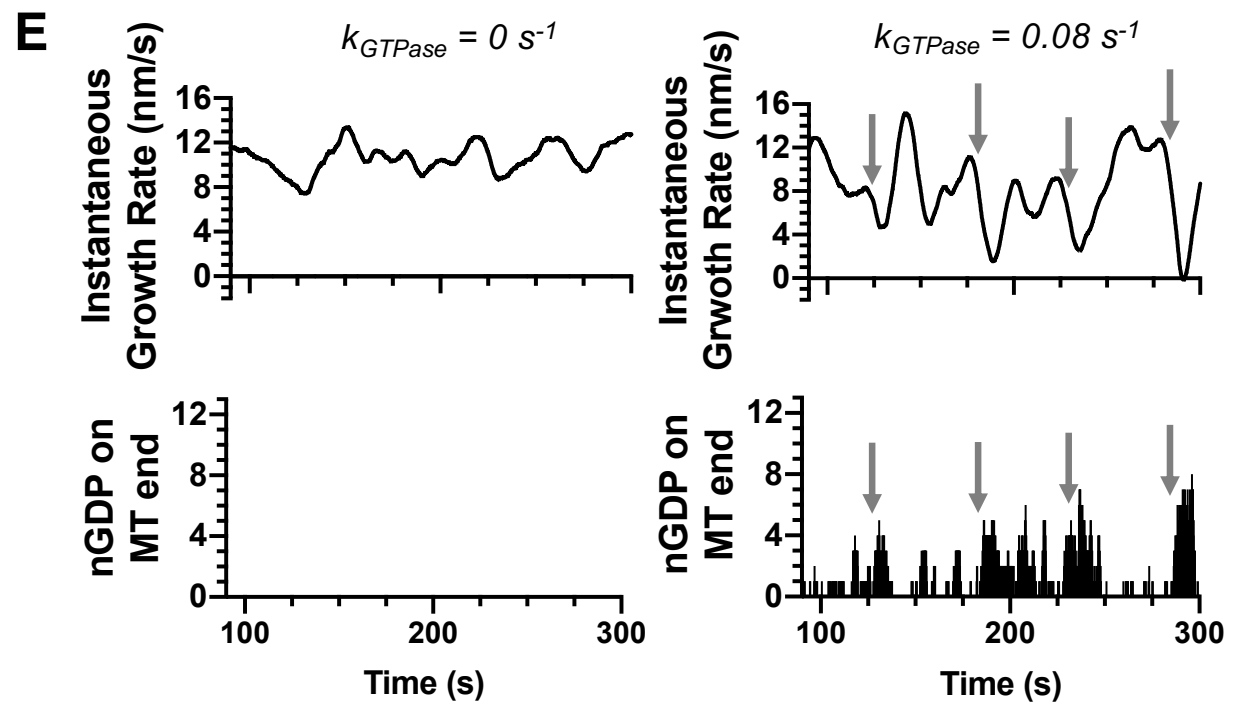

F

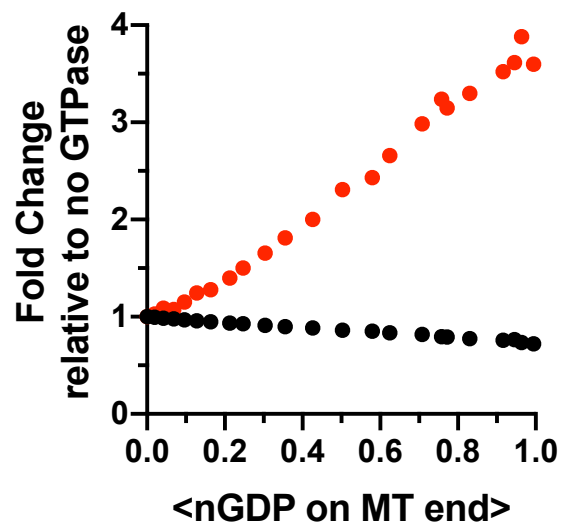


Figure 4. Exposure of GDP-tubulin on protofilament ends in simulations explains the excess fluctuations observed in GTP.

(A) Simulated growth rates for microtubules in GTP (blue) provide a good match to measured values (black; data from Fig. 3). The GTP simulations used the optimal on-rate constant determined for GMPCPP tubulin ( $\mathrm{k}_{\mathrm{on}}: 0.74 \mu \mathrm{M}^{-1} \mathrm{~s}^{-1}$; Fig. 2) and used corner and longitudinal affinity values $\left(\mathrm{K}_{\mathrm{D}}{ }^{\text {corner }}=2.9 \mu \mathrm{M} ; \mathrm{K}_{\mathrm{D}}{ }^{\text {long }}=1.7 \mathrm{mM}\right)$ optimized to fit the experimental growth rates in GTP.

(B) Fluctuations around the average growth rates in simulations (blue) substantially underestimate the measured values (black; data from Fig. 3).

1038

(C) Cartoon illustrating how GTPase activity is incorporated into the model. Two additional parameters are required: a rate constant for GTP hydrolysis, and a scale factor to model the 'weakening' effect of GDP on the longitudinal interface.

(D) Results from simulations at $12.5 \mu \mathrm{M} \alpha \beta$-tubulin (interaction strengths as in panel A and B) including GTPase activity. As the rate of GTPase increases in simulations, growth rates (black) decrease, whereas fluctuations in growth rate (red) increase to the point that they are comparable to the measured value (dotted line).

(E) Exposure of GDP on the growing end of the microtubule coincides with larger fluctuations in growth rate. In the absence of GTPase (left plots), the instantaneous growth rate varies in a relatively narrow range (top) and there is no GDP exposed on the microtubule end (bottom). When GTPase activity is present (right plots), the instantaneous growth rate explores a larger range including much slower values (top), and these especially slow growth rates coincide with exposure of one or more GDPterminated protofilaments (bottom).

(F) Dependence of growth rate (black) and growth fluctuations (red) on the average number of GDP exposed on the growing microtubule end. Values are plotted as fold change relative to the no GTPase condition. Fluctuations respond more strongly to the number of exposed GDP than do growth rates. 
Figure 4 - Figure Supplement 1

A

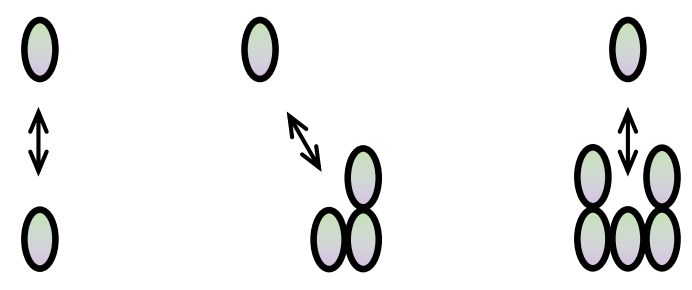

B

Site-independent association rate:

$$
\mathrm{k}_{\text {on }} \text { Long }=\mathrm{k}_{\text {on }} \text { Corner }=\mathrm{k}_{\text {on }} \text { Bucket }
$$

Site-dependent association rate:

$$
\begin{aligned}
\mathrm{k}_{\text {on }} \text { Long } & =1.7^{*} \mathrm{k}_{\text {on }} \text { Corner } \\
\mathrm{k}_{\text {on }} \text { Bucket } & =0.23^{*} \mathrm{k}_{\text {on }} \text { Corner }
\end{aligned}
$$

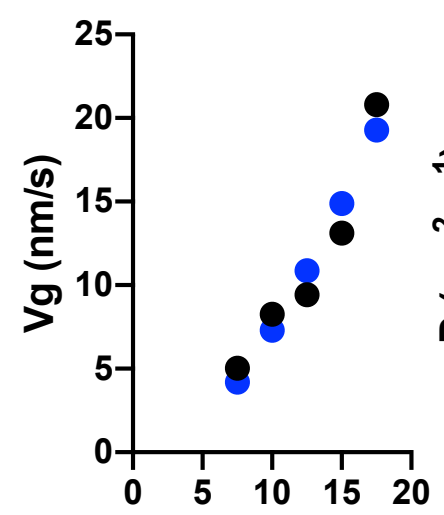

[ $\alpha \beta$-tubulin] ( $\mu \mathrm{M})$

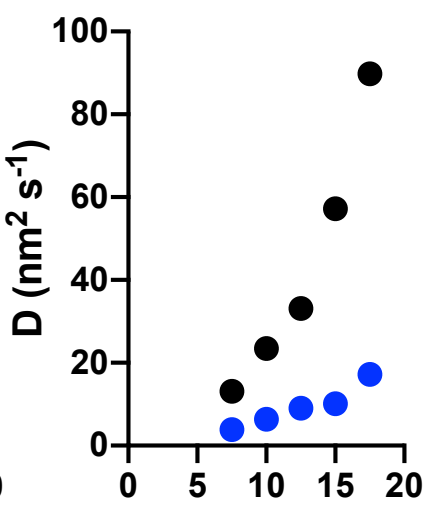

[ $\alpha \beta$-tubulin] ( $\mu \mathrm{M})$

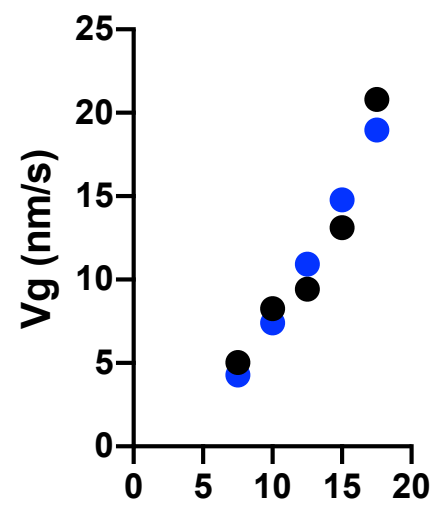

[ $\alpha \beta$-tubulin] ( $\mu \mathrm{M})$

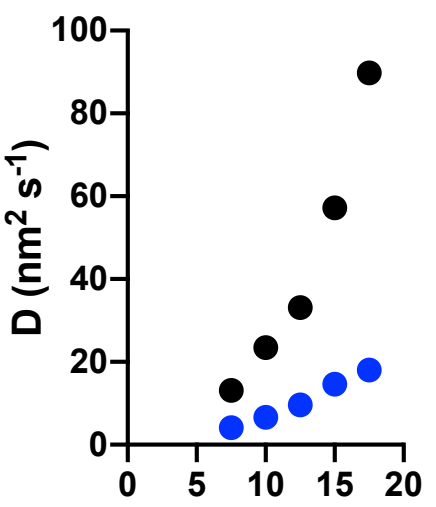

[ $\alpha \beta$-tubulin] $(\mu \mathrm{M})$ 
1058 Figure 4 - figure supplement 1. Site-dependent association rates do not explain the larger 1059 growth fluctuations observed in GTP.

(A) Cartoon of interactions occurring at the microtubule end. The interactions are ordered based on the number of lateral contacts: longitudinal $(n=0)$, corner $(n=1)$, and bucket $(n=2)$.

(B) Comparison between measured (black) and simulated (blue) growth rates (left) and fluctuations (right). For these panels, the model used one single association rate constant for longitudinal, corner, and bucket sites. The model was fit to growth rates only.

(C) Comparison between measured (black) and simulated (blue) growth rates (left) and fluctuations (right). For these panels, the model used site-dependent association rate constants for longitudinal, corner, and bucket sites. The relative factors relating the different rate constants were taken from (Castle and Odde, 2013). The model was fit to growth rates only. protofilaments and discretized into $0.5 \mathrm{~s}$ intervals. 
Figure 5

\section{GTPase activity inhibited}

1. Small concentration-dependent fluctuations

2. Variable end structures result in higher fluctuations

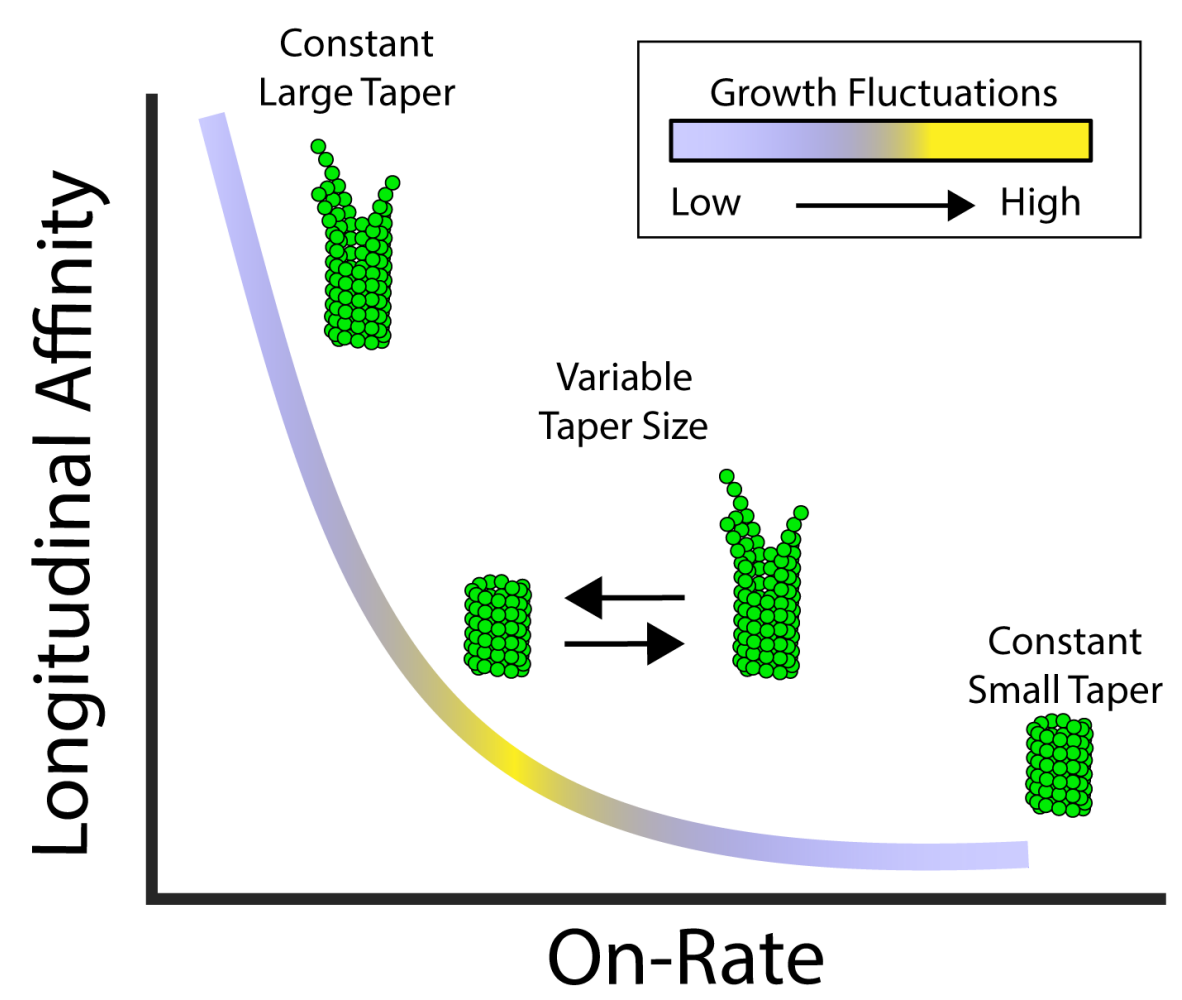

\section{GTPase activity present}

1. Large concentration-dependent fluctuations

2. Growth rate slows as terminal GDP-tubulin are exposed

\section{Exposure of GDP-tubulin}

at microtubule end

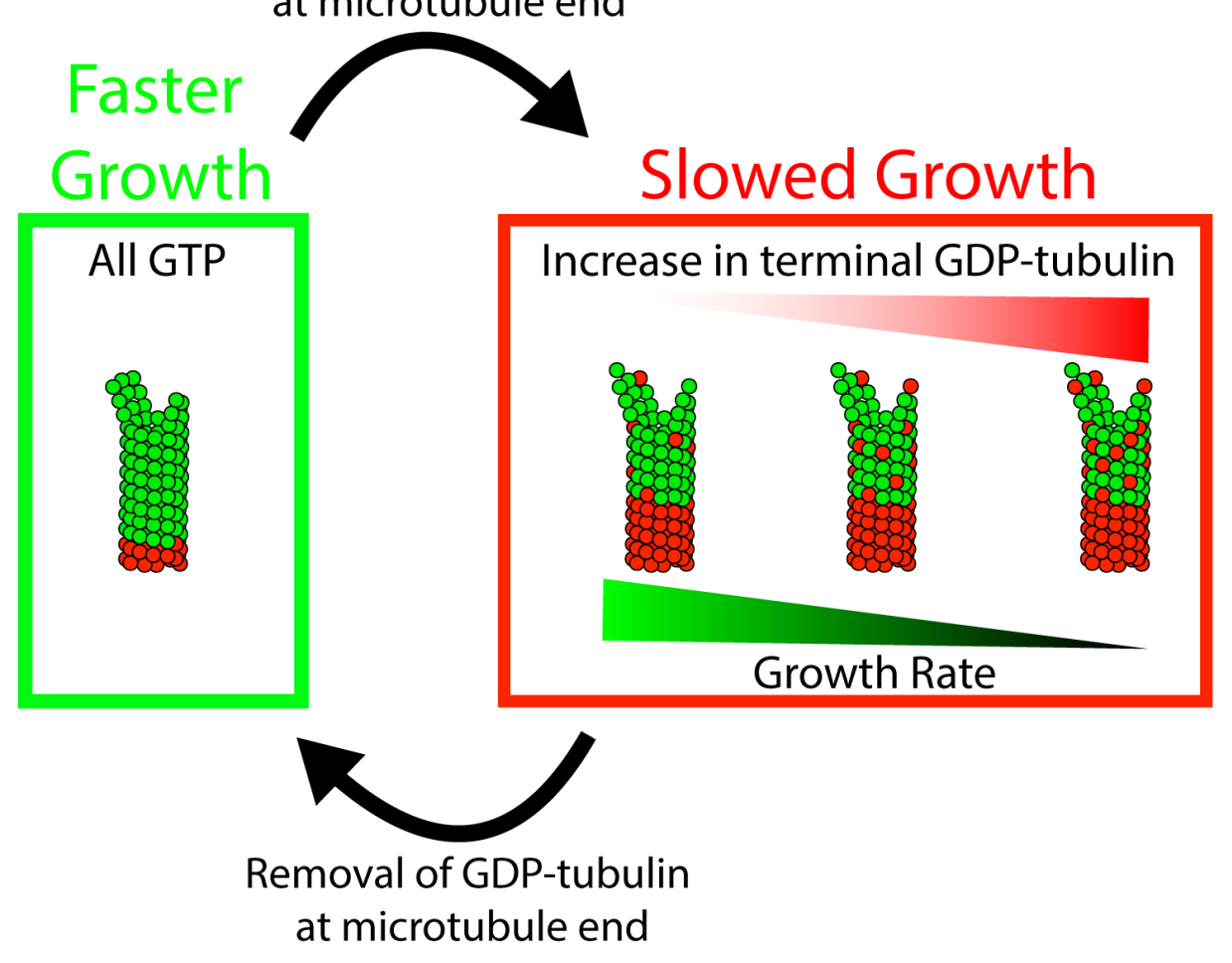




\section{Figure 5. Summary of results and insights}

1077 (left) Microtubule growth in the absence of GTPase activity can be accounted for by a simple

1078 biochemical model that incorporates the tubulin on-rate and the longitudinal affinity of the

1079 terminal tubulin at the plus-end. A slow on-rate and strong longitudinal affinity produces small

1080 fluctuations in growth and a large taper because the protofilaments grow somewhat

1081 independently of one another. A fast on-rate and weak longitudinal affinity produces small

1082 fluctuations in growth and a small taper because only corner interactions contribute to productive

1083 growth. The middle range produces fluctuations in both the growth rate and the size of the taper,

1084 which best match the experimental results.

1085

1086 (right) When GTPase activity is present, the growth fluctuations are much larger than in

1087 GMPCPP where hydrolysis is absent. The growth rate fluctuates more drastically due to

1088 switching between fast growth phases, when all terminal tubulin contain GTP, and slow growth

1089 phases resulting from one or more exposed GDP tubulin at the tip. When the exposed GDP

1090 tubulin either dissociates or is buried by an incoming GTP tubulin, an all-GTP tip with a

1091 corresponding fast growth rate is restored. 